\title{
Identification of imprinted genes subject to parent-of-origin specific expression in Arabidopsis thaliana seeds
}

Peter C McKeown ${ }^{1 \dagger}$, Sylvia Laouielle-Duprat ${ }^{1 \dagger}$, Pjotr Prins $^{2 \dagger}$, Philip Wolff ${ }^{3,4}$, Marc W Schmid ${ }^{5}$, Mark TA Donoghue ${ }^{1}$ Antoine Fort', Dorota Duszynska', Aurélie Comte ${ }^{1}$, Nga Thi Lao ${ }^{1}$, Trevor J Wennblom ${ }^{6}$, Geert Smant ${ }^{2}$, Claudia Köhler $^{3,4}$, Ueli Grossniklaus ${ }^{5}$ and Charles Spillane ${ }^{1^{*}}$

\begin{abstract}
Background: Epigenetic regulation of gene dosage by genomic imprinting of some autosomal genes facilitates normal reproductive development in both mammals and flowering plants. While many imprinted genes have been identified and intensively studied in mammals, smaller numbers have been characterized in flowering plants, mostly in Arabidopsis thaliana. Identification of additional imprinted loci in flowering plants by genome-wide screening for parent-of-origin specific uniparental expression in seed tissues will facilitate our understanding of the origins and functions of imprinted genes in flowering plants.

Results: CDNA-AFLP can detect allele-specific expression that is parent-of-origin dependent for expressed genes in which restriction site polymorphisms exist in the transcripts derived from each allele. Using a genome-wide cDNAAFLP screen surveying allele-specific expression of 4500 transcript-derived fragments, we report the identification of 52 maternally expressed genes (MEGs) displaying parent-of-origin dependent expression patterns in Arabidopsis siliques containing F1 hybrid seeds (3, 4 and 5 days after pollination). We identified these MEGs by developing a bioinformatics tool (GenFrag) which can directly determine the identities of transcript-derived fragments from (i) their size and (ii) which selective nucleotides were added to the primers used to generate them. Hence, GenFrag facilitates increased throughput for genome-wide CDNA-AFLP fragment analyses. The 52 MEGs we identified were further filtered for high expression levels in the endosperm relative to the seed coat to identify the candidate genes most likely representing novel imprinted genes expressed in the endosperm of Arabidopsis thaliana. Expression in seed tissues of the three top-ranked candidate genes, ATCDC48, PDE120 and MS5-like, was confirmed by Laser-Capture Microdissection and GRT-PCR analysis. Maternal-specific expression of these genes in Arabidopsis thaliana F1 seeds was confirmed via allele-specific transcript analysis across a range of different accessions. Differentially methylated regions were identified adjacent to ATCDC48 and PDE120, which may represent candidate imprinting control regions. Finally, we demonstrate that expression levels of these three genes in vegetative tissues are MET1-dependent, while their uniparental maternal expression in the seed is not dependent on MET1.
\end{abstract}

Conclusions: Using a CDNA-AFLP transcriptome profiling approach, we have identified three genes, ATCDC48, PDE120 and MS5-like which represent novel maternally expressed imprinted genes in the Arabidopsis thaliana seed. The extent of overlap between our cDNA-AFLP screen for maternally expressed imprinted genes, and other screens for imprinted and endosperm-expressed genes is discussed.

\footnotetext{
* Correspondence: charles.spillane@nuigalway.ie

† Contributed equally

${ }^{1}$ Genetics and Biotechnology Lab, Botany and Plant Science, National University of Ireland Galway (NUIG), C306 Aras de Brun, University Road,

Galway, Ireland

Full list of author information is available at the end of the article
}

\section{Biomed Central}

(C) 2011 McKeown et al; licensee BioMed Central Ltd. This is an Open Access article distributed under the terms of the Creative Commons Attribution License (http://creativecommons.org/licenses/by/2.0), which permits unrestricted use, distribution, and reproduction in any medium, provided the original work is properly cited. 


\section{Background}

Flowering plant (angiosperm) seeds are chimeric structures which contain tissues whose cells have unequal genomic contributions from the maternal and paternal parents [1-3]. Within Arabidopsis thaliana seeds the diploid embryo is comprised of cells containing nuclear genomes inherited equally from the maternal and paternal parents. In contrast, the triploid endosperm contains two maternally inherited nuclear genomes and one paternal genome. In addition, these two fertilisation products are surrounded by a maternally derived diploid seed coat [4]. The triploid endosperm is a terminally differentiated structure which nourishes the developing embryo, while the diploid maternal seed coat plays key roles in supporting the development of the seed and the embryo it harbours [5]. The interactions between these different tissues and genomes during seed development in plants remain poorly understood [6,7], despite the fundamental economic importance of angiosperm seeds. For any given gene, the relative and absolute contribution of each seed tissue to overall transcript levels in the seed can be difficult to determine.

An important consequence of the unequal contributions of male and female genomes to the chimeric seed is that seed development can be affected by genome dosage and parent-of-origin effects $[6,8,9]$. Such maternal effects include sporophytic maternal effects from the maternally derived seed coat and gametophytic maternal effects derived from the female gametes. Gametophytic maternal effects on seed development can be due (a) to general dosage effects in the endosperm; (b) to deposition of maternal transcripts expressed prior to fertilization in the egg and central cell that give rise to the embryo and endosperm, respectively; or (c) to epigenetic regulation of genes via genomic imprinting, whereby autosomal genes are uniparentally expressed post-fertilisation in a parent-of-origin-specific manner $[9,10]$.

Genomic imprinting has been predominantly described in mammals and flowering plants where it occurs in nutritive tissues (endosperm, placenta) and the developing embryo, although the latter is rare in plants [11]. While there are many theories regarding the evolution of genomic imprinting in mammals and plants, some focus on imprinting arising due to a 'parental conflict' over resource allocation $[12,13]$ or due to a necessity to limit gene dosage of key genes during early development [14,15].

Many imprinted genes (i.e. hundreds, typically arranged in gene clusters along chromosomes) have been identified and intensively studied in mammalian species [16]. Until recently (2010), only 18 imprinted genes had been reported across all flowering plant species, 11 of them in Arabidopsis thaliana (Additional file 1 Table S1). Imprinted genes have been identified using a range of different strategies, including: mutant screens for maternally-controlled seed abortion (Arabidopsis thaliana MEA and FIS2 [17]); screens for genes regulated by the FIS Polycomb group complex (Arabidopsis thaliana PHE1 [18]); microarray analyses searching for genes showing similar responses to known imprinted genes (Arabidopsis thaliana MPC [19]); endosperm mRNA profiling (maize nrp1 [20]), and via a combination of microarray profiling and allele-specific expression analysis on endosperm from reciprocally crossed inbred lines (eight maize genes [21]). Using $c d k a ; 1$ fertilized seeds which lack a paternal genome contribution to the (unfertilised) central cell, Shirzadi et al (2011) used microarray profiling to identify AGL36 as a maternally expressed imprinted gene amongst the 600 genes differentially regulated in the absence of a paternal genome [22]. The advent of next generation sequencing based transcriptomics has facilitated the recent identification of additional imprinted gene candidates in Arabidopsis thaliana seeds [23,24]. Hsieh et al (2011) [24] identified 43 confirmed imprinted genes ( 9 paternally expressed, 34 maternally expressed) in F1 hybrid seeds (7-8 days after pollination) from Ler-0 $\times$ Col-0 reciprocal crosses. Again using next generation sequencing approaches, Wolff et al (2011) [23] have identified 65 candidate imprinted genes in F1 hybrid seeds (4 days after pollination) from Bur- $0 \times$ Col- 0 reciprocal crosses of which 19 were confirmed in both cross directions (8 paternally expressed, and 11 maternally expressed). Hence, 'next generation' sequencing studies are now being employed to identify putative imprinted genes [23,24].

An indirect approach for the identification of novel imprinted genes has been conducted based on identification of differentially methylated regions (DMRs) as candidate imprinting control regions (ICRs) [25]. Genes acting as modifiers of genomic imprinting have also been identified in plants and include MET1 [26], DDM1 [17] and $D M E$ [27]. For example, the 5-methylcytosine DNA glycosylase gene $D M E$ is preferentially expressed in the central cell of the female gametophyte and can regulate the expression of some imprinted genes in the endosperm through demethylation of their ICRs [27]. In mutant $d m e$ endosperm ICRs remain methylated and as a result some imprinted genes are misregulated, which facilitates their detection [27].

While there are a number of genome-wide profiling approaches that can be used to identify allele-specific expression, there are several significant challenges for the definition of novel imprinted genes [28]. To distinguish between allele-specific expression effects that are either parent-of-origin dependent (e.g. imprinting) or independent, it is necessary to demonstrate the parentof-origin dependency of uniparental expression at 
imprinted loci by analysis of reciprocal F1 hybrid offspring. Furthermore, where maternal-specific expression is detected in a plant seed, it is necessary to distinguish between seed coat versus endosperm (and/or embryo) expression, and also to distinguish between transcripts maternally deposited in the egg and/or central cell versus transcripts generated post-fertilisation in the developing endosperm and/or embryo [11]. While imprinted genes displaying clear mutant phenotypes (e.g. medea) on seed development can facilitate interpretation of such loci as imprinted [10], many of the imprinted genes identified to date do not display any obvious mutant phenotype in seeds [29]. In some instances, promoter:reporter constructs have been used to identify cisregulatory regions that are required for imprinting $[19,30]$, while only one study has demonstrated post-fertilisation nascent uniparental de novo transcription of an imprinted gene in the endosperm [17].

The choice of transcript profiling platform is an important consideration for identification of novel imprinted genes. Microarrays are dependent on genes being expressed at a level sufficient to be detectable via hybridization and complementary strategies are necessary to also detect imprinted genes that may be lowly expressed. Hence, in this study we chose cDNA-AFLP [31] for genome-wide screening for novel imprinted genes. Although an early generation transcript profiling technology, as a PCR-based technology, cDNA-AFLP allows the amplification of even lowly expressed transcripts and can identify uniparentally expressed transcripts for all cases where there is a restriction site polymorphism between the parental alleles. To facilitate genome-wide cDNA-AFLP expression profiling, we have developed a gene-identifying bioinformatic software program, GenFrag, which can determine the identity of genes displaying parent-of-origin specific cDNA-AFLP expression profiles.

Our analysis of allele-specific expression of 4500 transcript-derived fragments (TDFs) in an experimental design based on the generation of reciprocal F1 hybrids seeds allowed the identification of 52 genes displaying maternal-specific expression (MEGs). The maternal specific expression of some of these MEGs may be due to genomic imprinting. Within these 52 maternally expressed genes, 18 represent genes that display higher relative and absolute expression levels in the endosperm relative to the maternal seed coat. Hence, the detection of maternal-specific expression of such genes in F1 hybrid seeds 4 days after pollination (dap) is consistent with such genes being subject to genomic imprinting in the developing endosperm. Four of these 18 MEGs have proximal differentially methylated regions (DMRs) in seed endosperm from wild-type and dme mutant backgrounds that may represent candidate imprinting control elements (ICRs). For the three top ranked candidates (ATCDC48, PDE120 and MS5-like) we confirm maternal-specific expression in F1 hybrid seeds 4 dap and characterise the control of their allele-specific expression at different developmental stages, and in different genetic and mutant backgrounds. Overall, we have identified a range of novel MEGs in Arabidopsis thaliana seeds, from which we further demonstrate that three are novel maternally expressed imprinted genes in Arabidopsis thaliana seeds.

\section{Results}

\section{CDNA-AFLP expression profiling of Arabidopsis thaliana} siliques containing F1 hybrid seeds detects 93 uniparentally-expressed TDFs

To identify genes which are uniparentally expressed in F1 hybrid seeds within siliques of Arabidopsis thaliana we employed a genome-wide cDNA-AFLP transcriptome profiling approach. At 3, 4 and 5 dap, RNA samples were generated from siliques containing F1 hybrid seeds generated via reciprocal crosses between the accessions Col-0 and Ler-0. These three stages correspond to developmental stages from the late globular (3 dap) to early and late heart stages ( 4 and 5 dap) of embryo development within the seed. These stages of embryo development were chosen to mitigate against the possibility of detection of maternally deposited longlived RNAs in the egg cell and/or central cell, and also because zygotic expression from both parental alleles is evident at these developmental stages [32]. In these samples, maternally expressed genes may be detected from either the silique or F1 seed tissues, and within the F1 seeds from either the maternal seed coat or the fertilisation products (i.e. the embryo and/or endosperm).

AFLP was performed on cDNA derived from RNA samples following restriction digestion with a frequently cutting enzyme (Bst YI) and a rare cutting enzyme (MseI) (Additional file 2 Figure S1). Fragments were ligated with adapters complementary to the restriction sites of the enzymes. To reduce the complexity of the mixture of fragments, a series of PCR amplifications were performed to generate subsets of fragments using selective primers. These selective primers share a common sequence, which corresponds to the adapters and a section of the restriction sites but are differentiated by one or two additional nucleotides at the 3'end, called selective nucleotides (Methods; Additional file 2 Figure S1).

The cDNA-AFLP generated transcript derived fragments (TDFs) were run on an ABI3130xl capillary analyser and visualized with fluorescently labelled probes to accurately estimate their size (see Methods). A total of 10,200 TDFs were detected across the three time points (3, 4, 5 dap). The TDFs ranged in size from 50 to 500 
base pairs (bp) and an average of $80 \mathrm{bp}$ was visualized per sample. Of the 10,200 TDFs screened, 4500 showed a polymorphism between cDNA derived from the reciprocal crosses between the two different accessions (genetic backgrounds) with sizes ranging from $100 \mathrm{bp}$ to 500 bp. Maternally expressed alleles were found in approximately equal numbers when each of the two accessions were used as the maternal parent in a reciprocal cross (Additional file 3 Table S2). For example, at the 4 dap time-point, 366 maternally expressed Col-0 alleles were detected in the Col- $0 \times$ Ler- 0 cross, while 306 maternally expressed Ler-0 alleles were detected in the reciprocal Ler- $0 \times$ Col- 0 cross. The numbers of maternally expressed TDFs detected were similar across the three developmental stages indicating consistency of maternal-specific transcription during early silique development. For each polymorphic allele (i.e. Col-0 vs Ler-0 alleles differing in a restriction site), only one fragment is detectable from each restriction digestion event as only those TDFs proximal to the poly-A tail were isolated for analysis. Hence for each of the two accessions there is no redundancy within the number of TDFs detected at each time-point.

To identify uniparentally expressed genes, cDNA-AFLP profiles for these 4500 polymorphic TDFs were compared between those obtained from siliques containing reciprocal $\mathrm{F} 1$ hybrid seeds (i.e. F1 progeny of Ler- $0 \times$ Col- 0 versus Col- $0 \times$ Ler- 0 crosses) and those obtained from the equivalent cross between plants of the same accession (i.e. Col- $0 \times$ Col- 0 , Ler- $0 \times$ Ler- 0 ). The samples at 3, 4 and 5 dap were used to filter for TDFs which displayed uniparental expression for at least two of the stages sampled. This strategy allowed the identification of 93 uniparentally expressed TDFs. All 93 of the uniparentally expressed TDFs displayed a maternal-specific expression pattern (Additional file 4 Table S3).

\section{Direct identification of genes based on TDF size and the selective nucleotides of each primer combination using the GenFrag bioinformatics program}

To identify the genes that produced the maternal TDFs detected in Arabidopsis thaliana siliques containing F1 hybrid seeds (Additional file 4 Table S3), we developed a bioinformatics program called GenFrag. GenFrag is designed to allow in silico identification of sequences of TDFs produced by cDNA-AFLP using publicly available cDNA and EST libraries (which for the well annotated Arabidopsis thaliana genome also includes all curated alternative splice variants [33]). Using these resources, GenFrag is designed to simulate the steps of the cDNAAFLP in silico by scanning existing Arabidopsis thaliana genome information for dual restriction enzyme cutting sites (see Methods and Additional file 2 Figure S1). Given the fragment size (as assessed on the capillary sequencer) and the selective nucleotides added to the primers used to generate the TDF, GenFrag can identify the corresponding sequence of a TDF and thereby the identity of the gene corresponding to the TDF. The GenFrag software is developed as open source software and is freely available for use online at: http://www.nem.wur.nl/UK/Research/bio/.

\section{GenFrag-based identification of 52 genes from the set of 93 maternally expressed TDFs}

GenFrag was used to identify genes corresponding to the 93 maternal specific TDFs (Additional file 4Table S3). To increase selectivity, we incorporated an option into GenFrag to only return the last matched fragment in a 5'-3' sequence i.e. the fragment closest to the poly-A tail of the mRNA. We combined this adaptation with a stringent range of $1 \mathrm{bp}$ deviation between the observed size of the TDF when run on the capillary analyser and the size predicted in silico for a candidate sequence. Using these conditions, GenFrag was able to determine unique sequence (i.e. gene ID) matches for 52 of the 93 maternally expressed TDFs identified (i.e. TDFs 1-52 in Additional file 4 Table S3). Of the remaining TDFs, 21 matched sequences shared by more than one gene and therefore could not be uniquely distinguished (TDFs 53-73 in Additional file 4 Table S3), while the remaining 20 could not be matched to any genes using the GenFrag approach (TDFs 74-93 in Additional file 4 Table S3). The lack of identification of these 20 TDFs may be due to aberrant enzyme restriction and/or incomplete coverage of the Arabidopsis thaliana transcriptome. The 52 unique sequence TDFs were matched to genes by BLAST searching the Arabidopsis thaliana genome (TAIR v.8). This allowed us to unambiguously identify 52 maternally expressed genes in Arabidopsis thaliana siliques containing F1 hybrid seeds (Table 1). Gene Ontology enrichment analysis of the 52 maternally expressed genes did not reveal any significant enriched terms (data not shown). Our set of 52 MEGs did not include the known imprinted genes from Arabidopsis thaliana, however, this is not surprising as most of these 52 MEGs have few SNP differences between the alleles from different accessions, and where they do, the SNPs do not disrupt the restriction sites that are scanned by the cDNA-AFLP technique using these restriction enzymes (Additional file 5 Table S4). For instance, there are no Col-0/Ler-0 SNPs in the coding sequence of the maternally expressed imprinted gene $M E D E A$. The 52 genes we identify represent novel maternally expressed genes (MEGs).

18 candidate imprinted genes in which the observed maternal expression is predominantly derived from higher transcript levels in the endosperm relative to the maternal seed coat

The 52 maternally expressed genes (MEGs) were detected in siliques containing reciprocal $\mathrm{F} 1$ hybrid 
Table 152 genes are identified as maternally expressed by GenFrag analysis of CDNA-AFLP TDFs sizes and the selective nucleotides of the primer combinations used to generate the TDFs

\begin{tabular}{|c|c|}
\hline Gene & Protein encoded \\
\hline At1g03070 & Glutamate binding protein \\
\hline At1g04700 & Protein kinase family protein \\
\hline At1g09390 & GDSL-motif lipase/hydrolase family protein \\
\hline At1g12420 & ACT Domain Repeat 8 (ACR8) \\
\hline At1g14880 & Unknown protein \\
\hline At1g16730 & Unknown protein \\
\hline At1g17840 & ABC transporter family protein \\
\hline At1g31820 & Amino acid permease family protein \\
\hline At1g54710 & AtATG18 \\
\hline At1g55320 & Ligase, similar to acyl-activating enzyme 17 (AAE17) \\
\hline At1g61990 & Mitochondrial transcription termination factor-related \\
\hline At1g65820 & Microsomal glutathione s-transferase, putative \\
\hline At1g73680 & Pathogen-responsive alpha-dioxygenase \\
\hline At1g74450 & Unknown protein \\
\hline At1g75680 & Arabidopsis thaliana glycosyl hydrolase 9B7 (ATGH9B7) \\
\hline At2g16480 & Unknown protein \\
\hline At2g21130 & Cyclophilin-like \\
\hline At2g26620 & Glycoside hydrolase family 28 protein \\
\hline At2g31510 & ARIADNE-like protein ARI7 (ARI7) \\
\hline At2g32000 & DNA topoisomerase family protein \\
\hline At2g36020 & Abscisic acid-responsive HVA22 family protein \\
\hline At2g40810 & $\begin{array}{l}\text { Arabidopsis thaliana homolog of yeast autophagy } 18 \mathrm{C} \\
\text { (ATG18c) }\end{array}$ \\
\hline At2g45315 & Unknown \\
\hline At3g09840 & Cell division cycle 48 (ATCDC48) \\
\hline At3g12370 & Mitochondrial RPL10 \\
\hline At3g20760 & Nse4, component of Smc5/6 DNA repair complex \\
\hline At3g22260 & $\begin{array}{l}\text { Ovarian tumor domain-like cysteine protease family } \\
\text { protein }\end{array}$ \\
\hline At3g24780 & Uncharacterised conserved protein \\
\hline At3g25530 & Gamma-hydroxybutyrate dehydrogenase (ATGHBDH) \\
\hline At3g29360 & UDP-glucose 6-dehydrogenase \\
\hline At3g47250 & Unknown protein \\
\hline At3g51280 & Similar to male sterility MS5 \\
\hline At3g55250 & Similar to calcium homeostasis regulator $(\mathrm{CHoR} 1)$ \\
\hline At3g57510 & Arabidopsis endo-polygalacturonase 1 (ADPG1) \\
\hline At3g59380 & FARNESYLTRANSFERASE A (FTA) \\
\hline At4g00180 & YABBY gene family member \\
\hline At4g01000 & Ubiquitin family protein \\
\hline At4g16830 & Nuclear RNA-binding protein (RGGA) \\
\hline At4g21270 & AT KINESIN 1 \\
\hline At4g29450 & Leucine-rich repeat protein kinase, putative \\
\hline At4g33450 & Myb domain protein 69 (AtMYB69) \\
\hline At4g37530 & Peroxidase, putative \\
\hline At5g04895 & ATP binding/helicase/nucleic acid binding protein \\
\hline
\end{tabular}

Table 152 genes are identified as maternally expressed by GenFrag analysis of cDNA-AFLP TDFs sizes and the selective nucleotides of the primer combinations used to generate the TDFs (Continued)

\begin{tabular}{ll}
\hline At5g16620 & $\begin{array}{l}\text { Pigment defective embryo (PDE120) chloroplast import } \\
\text { (Tic40) }\end{array}$ \\
\hline At5g17080 & Cathepsin-related protein \\
\hline At5g35730 & EXS family protein/ERD1/XPR1/SYG1 family protein \\
\hline At5g35737 & Unknown protein \\
\hline At5g38320 & Unknown protein \\
\hline At5g39510 & VESICLE TRANSPORT V-SNARE 11 (VTI11) \\
\hline At5g40390 & Seed imbibition 1 (SIP1) \\
\hline At5g61300 & Unknown protein \\
\hline At5g56310 & ATHB5 \\
\hline 52 maternally-expressed genes were identified from transcript-derived \\
fragments generated by cDNA-AFLP of hybrid A. thaliana siliques. 93 TDFs \\
were identified using GenFrag on the basis of their size and the selective \\
nucleotides of the primer combinations used to generate them. These were \\
matched to the 52 genes listed by BLASTN against $A$. thaliana genome (TAIR \\
v.8). Nine genes which have been reported as preferentially endosperm- \\
enriched (Day et al., 2008) are marked in bold.
\end{tabular}

seeds where the maternal-specific expression could be derived from the silique, the maternal seed coat, the endosperm and/or the embryo. Seed expressed genes which are predominantly maternally expressed in the endosperm from 3 dap (late globular stage embryos) are excellent candidates for regulation by genomic imprinting. It was recently shown that embryo development up to the globular stage does not depend on de novo transcription while endosperm development requires active transcription following fertilization, suggesting that maternally deposited RNAs do not play a predominant role in the endosperm [34]. Thus, mRNAs detected in the endosperm at $\geq 3$ dap are most likely to be derived from de novo transcription post-fertilization. To identify which of the 52 maternally expressed genes are predominantly expressed in the endosperm at high expression levels, we used a publicly available expression dataset (Seed Gene Network - Harada-Goldberg Arabidopsis Laser Capture Microdissection Gene Chip Data Set, http://seedgenenetwork.net; [35]) where the relative expression levels of genes in the seed coat and endosperm tissues (peripheral, chalazal and micropylar fractions) of seeds at the globular stage of embryo development (3 dap) have been assessed.

From the 52 maternally expressed genes, we could identify 32 genes which had strong signals of expression in the 3 dap seed. Eleven genes were not detected as they did not have probes in the array dataset used, or their probes also matched another gene. Nine genes were not expressed in seeds and therefore may be good candidates for silique specific MEGs. Comparing the expression levels between the endosperm and the seed 
coat, we found three MEGs which were exclusively expressed in the seed coat but no MEGs which were absent from the seed coat but were expressed in the endosperm. However, twenty-nine MEGs showed expression in both the endosperm and the seed coat. We considered that if maternal-specific expression can be demonstrated in seeds for MEGs where the majority of the expression level signal is from the endosperm, that such a pattern would be strongly indicative of a maternally expressed imprinted gene in the endosperm. Biallelic expression in the endosperm should also be easier to detect in such cases. Hence, for these twentynine MEGs, we aimed to identify genes where the majority of the expression detected in the seed is due to the endosperm fraction. We selected the 18 genes out of the 29 that showed higher expression in the endosperm compared to the seed coat and ranked these genes based on the absolute difference of expression levels between the highest expressing endosperm fraction and the seed coat (Table 2). We reasoned that genes displaying the highest levels of expression in the endosperm of 3 dap seeds were least likely to be genes where maternal-specific transcripts detected could be due to maternal deposition of transcripts in the central cell [34] or transferred from the maternal seed coat as has recently been proposed [24] i.e. we focussed on genes which are highly expressed in the endosperm relative to the maternal seed coat. As a complementary approach, we also compared these genes on the basis of relative transcription levels (Additional file 6 Table S5). For these MEGs with significantly higher expression levels in the endosperm when compared to the seed coat, maternal-specific expression detected in reciprocal F1 hybrid seeds at 4 dap is consistent with regulation via genomic imprinting in the endosperm. Using these approaches, we chose the three top ranked genes as measured by total enrichment of expression in the endosperm, ATCDC48 (At3g09840), PDE120 (At5g16620) and MS5-like (At3g51280) as our strongest imprinted candidates for further investigation. Although PDE120 and MS5-like were less highly expressed in the endosperm in total, they were also the most highly ranked genes as measured by ratio of endosperm:seed coat expression (Additional file 6 Table S5) and as

Table 2 Maternally expressed genes ranked by absolute expression level difference between highest-expressing endosperm fraction and seed coat

\begin{tabular}{|c|c|c|c|c|c|c|c|}
\hline Gene ID & $\begin{array}{l}\text { Seed coat } \\
\text { expression } \\
\text { level }\end{array}$ & $\begin{array}{c}\text { Embryo } \\
\text { expression } \\
\text { level }\end{array}$ & $\begin{array}{c}\text { Peripheral } \\
\text { endosperm } \\
\text { expression } \\
\text { level }\end{array}$ & $\begin{array}{c}\text { Micropylar } \\
\text { endosperm } \\
\text { expression } \\
\text { level }\end{array}$ & $\begin{array}{c}\text { Chalazal } \\
\text { endosperm } \\
\text { expression } \\
\text { level }\end{array}$ & $\begin{array}{c}\text { Absolute difference of } \\
\text { expression levels between } \\
\text { highest-expressing } \\
\text { endosperm fraction and seed } \\
\text { coat (hEF-SC) }\end{array}$ & $\begin{array}{c}\text { Ratio of expression levels } \\
\text { between highest- } \\
\text { expressing endosperm } \\
\text { fraction and seed coat } \\
\text { (hEF/SC) }\end{array}$ \\
\hline $\begin{array}{l}\text { At3g09840 } \\
\text { (AtCDC48A) }\end{array}$ & 9462.69 & 12859.55 & 8565.74 & 7199.95 & $15983.67 *$ & 6520.97 & 1.69 \\
\hline $\begin{array}{l}\text { At5g16620 } \\
\text { (PDE120) }\end{array}$ & 1882.19 & 3721.39 & $7328.89 *$ & 1547.72 & 594.65 & 5446.69 & 3.89 \\
\hline $\begin{array}{l}\text { At3g51280 } \\
\text { (MS5) } \\
\end{array}$ & 143.71 & 6909.54 & $3598.61 *$ & 425.12 & 170.39 & 3454.9 & 25.04 \\
\hline At4g16830 & 1403.41 & 2234.66 & 3777.26 * & 3520.61 & 1358.85 & 2373.85 & 2.69 \\
\hline At5g63330 & 364.44 & 215.12 & 512.38 & 340.48 & $1942.53 *$ & 1578.09 & 5.33 \\
\hline At1g73680 & 2286.18 & 68.31 & 1281.93 & 3787.4 * & 1095.28 & 1501.21 & 1.66 \\
\hline At1g03070 & 150.95 & 22.77 & 43.96 & 70.68 & $1273.49 *$ & 1122.55 & 8.44 \\
\hline At3g24530 & 839.73 & 1359.11 & 1940.84 * & 1502.07 & 352.68 & 1101.11 & 2.31 \\
\hline At1g65820 & 757.13 & 253.23 & 413.46 & $1813.32 *$ & 612.73 & 1056.2 & 2.39 \\
\hline At3g17000 & 416.34 & 137.53 & 165.76 & 440.71 & $1401.76 *$ & 985.42 & 3.37 \\
\hline At5g39510 & 1934.73 & 812.5 & 1357.99 & $2472.39 *$ & 2071.39 & 537.65 & 1.28 \\
\hline At1g25370 & 362.23 & 56.59 & 52.88 & 339.09 & 718.4 * & 356.17 & 1.98 \\
\hline At3g59380 & 333.7 & 299 & 481.63 & 597.3 & $631.7 *$ & 298.01 & 1.89 \\
\hline At3g55250 & 183.62 & 264.14 & $461.66 *$ & 219.78 & 83.6 & 278.04 & 2.51 \\
\hline At2g31510 & 398.3 & 455.28 & 416.09 & $642.15 *$ & 195.12 & 243.85 & 1.61 \\
\hline At2g16480 & 620.32 & 793.04 & $854.07 *$ & 611.2 & 577.62 & 233.75 & 1.38 \\
\hline At1g61990 & 265.14 & 479.11 & 335.01 & 403.18 & $470.95 *$ & 205.81 & 1.78 \\
\hline At2g32000 & 280.44 & 244.55 & 333.14 * & 176.92 & 98.83 & 52.7 & 1.19 \\
\hline
\end{tabular}

Expression levels in Arabidopsis thaliana seed coat (SC), embryo and peripheral, micropylar and chalazal endosperm tissues of 18 maternally expressed genes. * highlights the highest-expressing endosperm fraction (hEF). Microarray data is from Seedgenenetwork (Harada-Goldberg Arabidopsis Laser Capture Microdissection Gene Chip Data Set, http://seedgenenetwork.net). 
noted in Table 1 have previously been reported as preferentially endosperm-expressed in a microarray study performed by Day et al. [36]. Hence we consider all three of these MEGs to be principally expressed in the F1 endosperm relative to the maternal seed coat.

\section{Laser capture microdissection (LCM) and qRT-PCR confirm expression of ATCDC48, PDE120 and MS5-like in Arabidopsis thaliana seed}

To validate the expression patterns of the three top ranked imprinted gene candidates ATCDC48, PDE120 and MS5-like, we used Laser Capture Microdissection (LCM) to microdissect Arabidopsis thaliana seeds (5 dap) of accession Ler-0 into endosperm (ES), seed coat (SC) and embryo (EM) fractions. The three LCM tissues were screened by qualitative end-point RT-PCR to investigate tissue-specific expression of each gene within the seed at 5 dap, which confirmed that all three genes are indeed expressed in Arabidopsis thaliana seeds (Additional file 7 Figure S2). Transcripts were detected in both the seed coat and endosperm for all three genes, while ATCDC48 and MS5-like were also detected in the embryo. Although this qualitative RT-PCR analysis provided no indication of relative expression levels in each of the three distinct parts of the seed, it served to independently confirm that the three genes are indeed expressed in seed tissues at 5 dap in the tissues predicted by the Seed Gene Network expression database (Table 2).

To determine how the expression levels of these genes in seeds varied over the time-course covered by our cDNA-AFLP experiment, we performed qRT-PCR on seeds at different time-points 3, 4 and 5-6 days after manual pollination. The existing data for whole-seed expression levels in Ws-0 (Seed Gene Network, [35]) predicted that expression of MS5-like and CDC48A would increase through development (across globular, heart and elongated cotyledon stages). In our qRT-PCR analysis, we found that this expression pattern was conserved in both Col-0 and Ler-0 seeds (Figure 1A, B) indicating that for these genes there is little effect of accession background on total expression levels. However, we also found increased expression of PDE120 at the 5-6 dap time-point in both accessions, which differed from the Ws-0 data (Seed Gene Network) (Figure 1A, B).

To preclude any differences on expression levels that could be due to a hybrid background, we also measured expression of PDE120 within reciprocal Col-0 $\times$ Ler- 0 crosses at the 3, 4 and 5-6 dap time-points and again found increased expression through seed development (Figure 1C). This suggests that the expression patterns
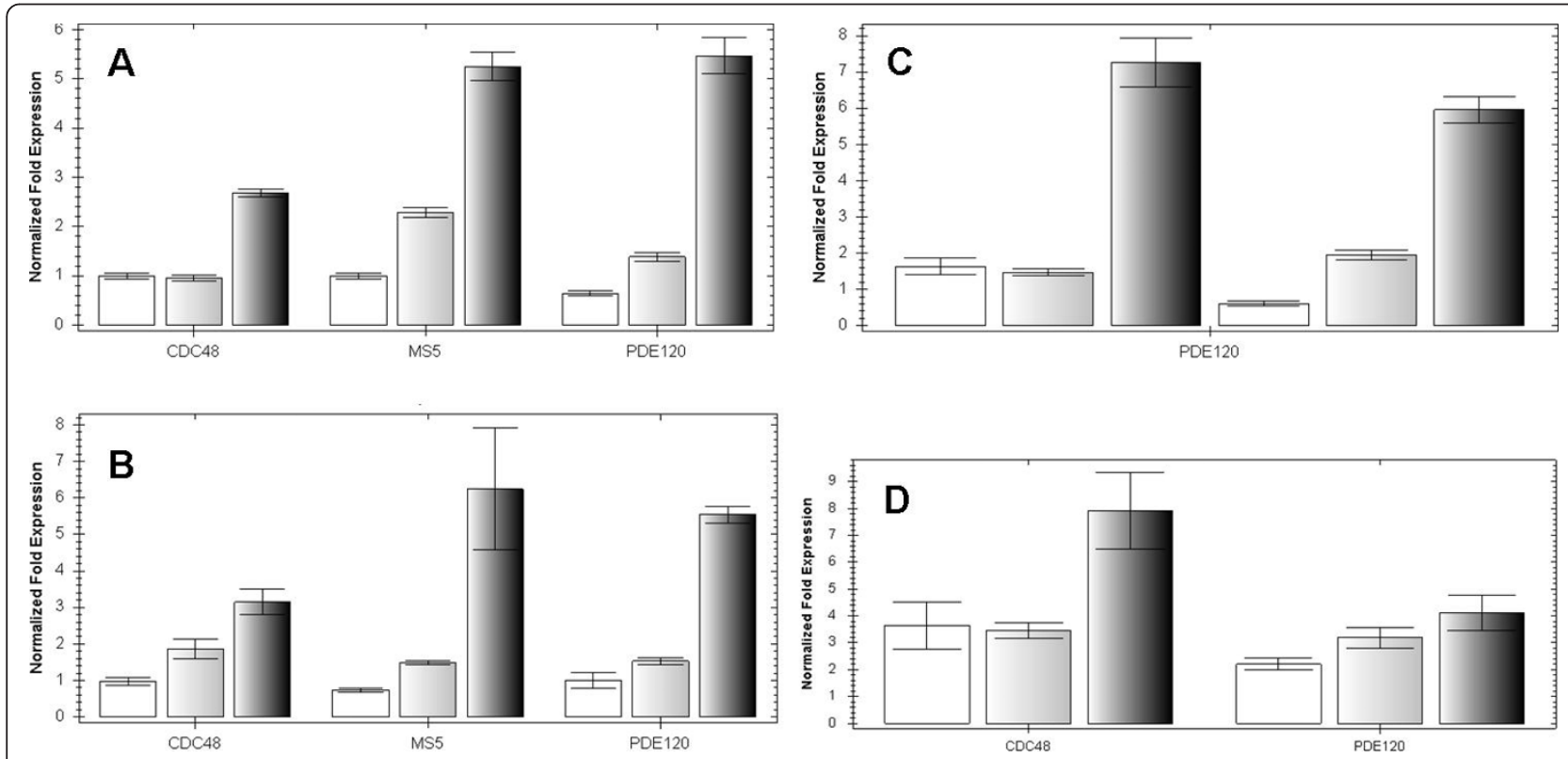

Figure 1 Expression profiles of candidate imprinted genes in Arabidopsis thaliana seed as determined by qRT-PCR. 1A. Expression of AtCDC48A, MS5-like and PDE120 increases though Col-0 seed development at 3 dap (left-hand columns), 4 dap (middle columns) and 5-6 dap (right-hand columns) 1B. Expression of AtCDC48A, MS5-like and PDE120 increases though Ler-0 seed development at 3 dap (left-hand columns), 4 dap (middle columns) and 5-6 dap (right-hand columns) 1C. PDE120 is expressed in hybrid seeds in similar patterns to non-hybrid seeds. Determined at 3, 4 and 5-6 dap for Col-0 $\times$ Ler-0 (first 3 columns) and Ler-0 $\times$ Col-0 (second three columns). 1D. AtCDC48A and PDE120 are expressed only at low levels in ovules of Col-0 (left-hand columns) or Ler-0 (middle columns) compared to Col-0 4 dap seed (right-hand columns). Standard errors are shown. 
of these three seed-expressed genes, which are similar in both parental accessions, are not significantly altered in their F1 hybrid offspring, although transcript levels of PDE120 might be slightly higher at 3 dap in the Col-0 $\times$ Ler-0 cross direction. Because expression increases throughout development, and was, in contrast, lower in pre-fertilized ovules (Figure 1D), this suggests that the expression we have detected is due to de novo post-fertilisation transcription and not maternal deposition of long-lived RNA transcripts from the central cell and/or egg cell to the post-fertilisation endosperm and/or embryo, respectively.

The maternally expressed seed genes ATCDC48, PDE120 and MS5-like are subject to gene-specific imprinting in different genetic backgrounds

Genomic imprinting can be 'gene-specific' (where all alleles of the gene are imprinted in the majority of genetic backgrounds) or 'allele-specific' (where only one or a few alleles are imprinted in specific genetic backgrounds) [28]. To validate the three top-ranked genes as maternally expressed imprinted genes and to test for gene- vs allele-specific imprinting, we identified SNPs in the coding regions of each gene between the Col- 0 and C24 accessions, and between the Col-0 and Bur-0 accessions. We sequenced cDNA from reciprocal F1 hybrid seeds (4 dap) to detect any evidence of mono-allelic expression patterns consistent with regulation of the genes by genomic imprinting. To confirm the effects in both of the genetic backgrounds used for cDNA-AFLP, we also sequenced SNPs in cDNA from F1 hybrid seeds (4 dap) of Ler-0 $\times$ Col-0 crosses for PDE120 and MS5like. In all cases, we found that ATCDC48, PDE120 and MS5-like were maternally expressed in F1 hybrid seeds at 4 dap (Figure 2; Additional file 8 Figure S3). While binary imprinted expression (on/off) was observed for ATCDC48 and PDE120, MS5-like displayed preferential expression of the maternally inherited allele (Figure 2). This indicates that the imprinted status of these three genes, like their expression levels (Figure 1), is conserved across divergent accessions and that they likely represent cases of gene-specific imprinting.

As a more general validation of the cDNA-AFLP approach to detect maternally expressed seed genes, we

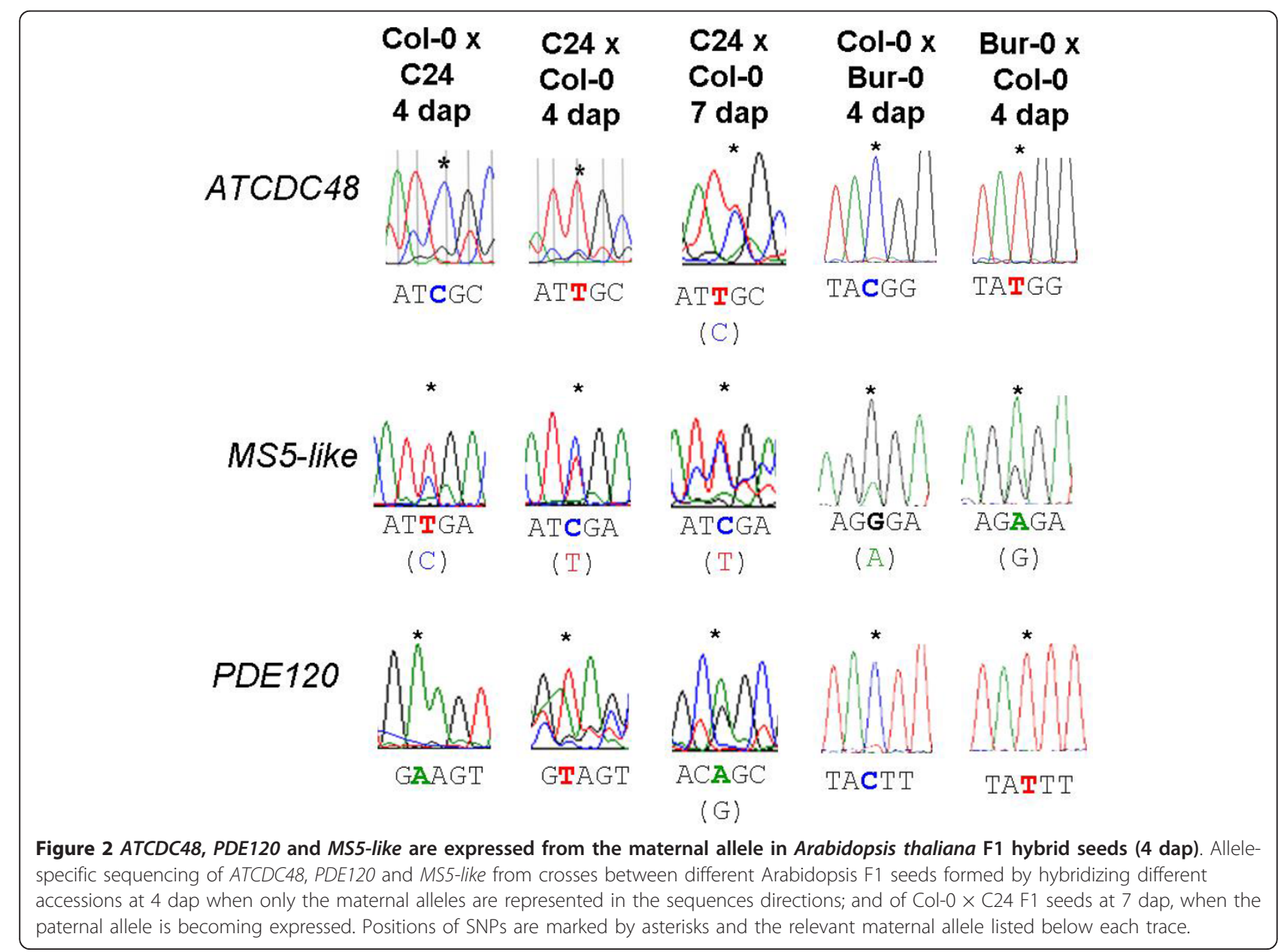


chose six further genes predicted to be expressed in seed tissues and sequenced SNPs in cDNA generated from Col-0 $\times$ C24 and C24 $\times$ Col-0 F1 hybrid seeds at 4 dap. In all six cases, we validated maternal-specific expression. We have therefore validated $9 / 52=17 \%$ of the genes identified as uniparentally expressed by cDNA-AFLP as MEGs (Additional File 9 Figure S4).

For the top ranked imprinted gene ATCDC48, we also quantified the extent of imprinting using Quantification of Allele Specific Expression by Pyrosequencing (QUASEP), a technique based on real-time pyrophosphate $\left(\mathrm{PP}_{\mathrm{i}}\right)$ detection [32-34], which allows precise relative quantification of SNP frequencies (Figure 3). QUASEP was performed on the maternally expressed imprinted gene ATCDC48 using cDNA collected from reciprocal Col-0 $\times$ C24 F1 hybrid seeds (4 dap). The known imprinted genes FWA and PHE1 were used as controls (Table 3), which confirmed maternal-specific (binary) and paternal-specific (preferential) expression patterns for these two imprinted genes, respectively [26,37]. $P H E 2$, the non-imprinted endosperm-expressed homologue of PHE1, was used as a biallelic control (Table 3). We found that in F1 hybrid seeds at 4 dap the relative expression level from the maternally inherited allele of ATCDC48 was $100 \%(\mathrm{Col}-0 \times \mathrm{C} 24)$ and $80.5 \%(\mathrm{C} 24 \times$ Col-0) indicating that $A T C D C 48$ displays maternal-specific expression (Figure 2). Although ATCDC48 is subject to expression in the seed coat, it displays high expression levels in the chalazal endosperm (Table 2), which is consistent with post-fertilisation transcription in the endosperm rather than a scenario of deposition of maternal transcripts in the central cell. Thus, the expression pattern of $A T C D C 48$ is consistent with $A T C D C 48$ being a novel maternally expressed imprinted gene in the endosperm of Arabidopsis thaliana seeds.

Both ATCDC48 and MS5-like also show high levels of expression in the embryo (Table 2). Biallelic expression at the heart stage of embryo development would be expected for most embryo-expressed genes, following the earlier reactivation of the paternal genome (from the globular embryo stage onwards) in Arabidopsis thaliana [32]. In the case of MS5-like, expression within the seed is largely confined to the embryo and to the peripheral endosperm. It is likely that imprinting of MS5-like occurs exclusively within the 4 dap endosperm whilst expression in the embryo is biallelic, which could explain the partial peak of expression from the paternal allele of this gene (Figure 2). For ATCDC48 however, the detection of almost exclusively maternal transcripts by sequencing and QUASEP could suggest that $A T C D C 48$ may undergo delayed reactivation of the paternally inherited allele in the 4 dap embryo.

\section{Expression of imprinted genes in endosperm of seeds at later developmental stages}

In a recent study, Hsieh et al. (2011) [24] screened for novel imprinted genes in 7-8 dap seed from reciprocal crosses between Col-0 and Ler-0. The differences

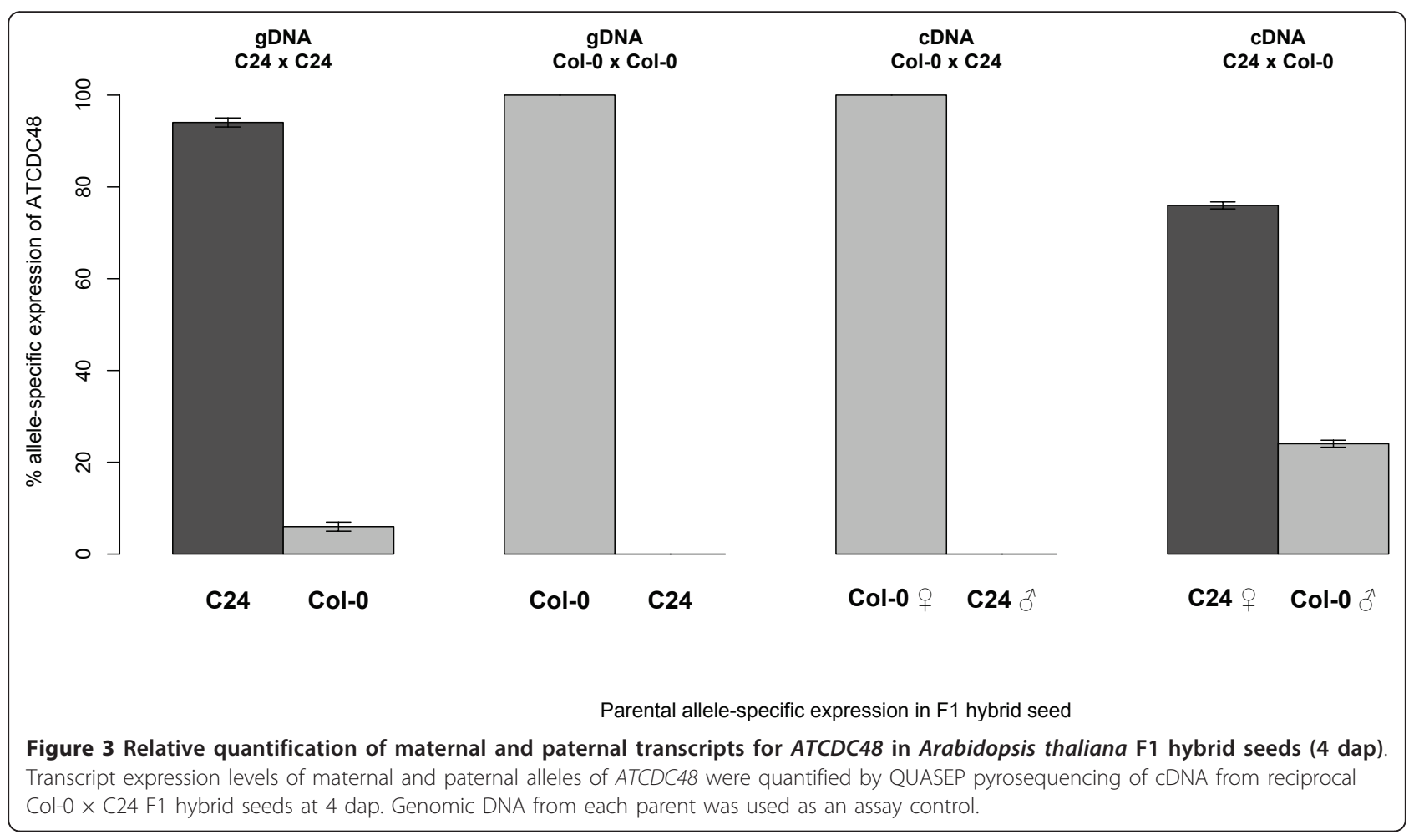


Table 3 Comparative controls for quantification of maternal expression of ATCDC48A by QUASEP.

\begin{tabular}{lllllll}
\hline Gene & Name & SNP & Col-0 allele (maternal) & C24/Ler-0 allele (maternal & Mean maternal & Imprinted status \\
\hline At3g09840 & CDC48A & Col-0/C24 & $100.0 \%$ & $80.5 \%$ & $90.4 \%$ & MEG test gene \\
\hline At4g25530 & FWA & Col-0/Ler-0 & $92.1 \%$ & $97.1 \%$ & $94.6 \%$ & MEG control gene \\
\hline At1g65330 & PHE1 & Col-0/Ler-0 & $27.4 \%$ & $12.8 \%$ & $21.1 \%$ & PEG control gene \\
\hline At1g65330 & PHE2 & Col-0/Ler-0 & $56.3 \%$ & $35.3 \%$ & $45.8 \%$ & Biallelic control \\
\hline
\end{tabular}

FWA and PHERES1 were used as maternally and paternally expressed controls, respectively; the non-imprinted gene, PHERES2 was as a control expressed from both alleles within the endosperm.

between the numbers of uniparental TDFs identified by cDNA-AFLP at 3, 4 and 5 dap (Additional file 2 Table S2), with only 92 uniparental TDFs detected at multiple developmental stages, suggests some temporal dynamism in the regulation of imprinting in Arabidopsis thaliana seeds which could potentially explain the lack of overlap between our results and those of Hsieh et al. [24]. To test this, we investigated whether the MEGs we had identified at 4 dap remained monoallelic or became biallelic at later developmental stages. Our results indicate that in cDNA from 7 dap seed, paternal alleles were more highly expressed than at 4 dap for all three of the genes (Figure 2). In the case of ATCDC48A, this rendered the expression fully biallelic, whilst the maternal allele was still preferentially expressed for MS5-like and PDE120 (Figure 2). At the 7 dap time-point, while all three genes are expressed from the embryo and endosperm, the relative and absolute contributions of each tissue to total transcript levels in the 7 dap seed are not known. Hence, the increased expression of the paternal allele observed in the 7 dap seed could arise from loss of imprinting and/or a shift in the relative proportion of embryo versus endosperm tissues amounts in the 7 dap seed (compared to the 4 dap seed). In the latter scenario, the MEG could remain imprinted in the endosperm tissue, but be masked by a biallelic expression signal from the more abundant embryo tissue at 7 dap. The expression of both alleles would be likely to preclude their identification at the $\mathrm{p}<0.001$ cut-off used for most gene identifications by Hsieh et al. [24]. We also considered the concordance between our dataset and a further next-generation sequencing screen performed by Wolff et al. [23] (Additional File 10 Figure S5) and found no overlap either with our screen or with that of Hsieh et al. [24] (see also Discussion). We also found very little overlap (seven out of 100) between imprinted genes detected by these two studies and differentially methylated regions (DMRs) previously predicted by Gehring et al. [25]. This prompted us to consider the possible existence of unidentified DMRs which could act as imprinting control regions (ICRs) associated with our imprinted genes.
Identification of DMRs at the ATCDC48, PDE120 and MS5like loci

While the imprinting control regions (ICRs) of imprinted genes in mammals often overlap with differentially methylated regions (DMRs), the genome-wide distribution of DMRs means that only some of these are likely to be ICRs [38-41]. In plant genomes, ICRs that coincide with DMRs have been identified for the imprinted genes FWA [26,42], PHE1 [30], and MPC [19]. As noted above, however, they have not been detected for many other imprinted genes, and induction of imprinting by many putative DMRs [11] remains unconfirmed (Additional File 10 Figure S5). Using available methylation data for wild-type and $d m e$ endosperm [43], we searched for DMRs in the genomic vicinity of the maternally expressed imprinted loci $A T C D C 48$, PDE120 and MS5-like.

We identified DMRs that could potentially act as ICRs for PDE120 and ATCDC48 (Figures 4A and 4B) by analysing expression data derived from endosperm of the wild type and endosperm of seeds deficient for a maternal $D M E$ allele [43]. These were retrieved from ArrayExpress and the percentage of methylation at cytosines situated between the genes immediately upstream and downstream of the gene bodies calculated. A DMR was located 432 bp downstream of ATCDC48A containing 26 cytosines, of which 6 are hypermethylated in dme (Figure 4A). Four DMRs were located upstream of PDE120 at distances of 8273 bp (30 cytosines, 17 hypermethylated in $d m e), 5377$ bp (49 cytosines, 6 hypermethylated in dme), 4620 bp (46 cytosines, 13 hypermethylated in dme) and 3635 bp (115 cytosines, 12 hypermethylated in dme) (Figure 4B). No obvious DMEdependent DMRs could be identified in the genomic neighbourhood of the imprinted gene MS5-like (Figure $4 \mathrm{C})$. We also analysed our entire portfolio of candidate imprinted genes (Table 2) for potential DMRs in their vicinity. In contrast to our three top ranked imprinted genes, we could only identify DMRs for two additional genes out of the other 49, namely At1g25370 (encoding a protein of unknown function containing a DUF1639) and At2g32000 (encoding a DNA topoisomerase, type 1A) (Additional File 11 Figure S6). Overall, these data 


\section{A}

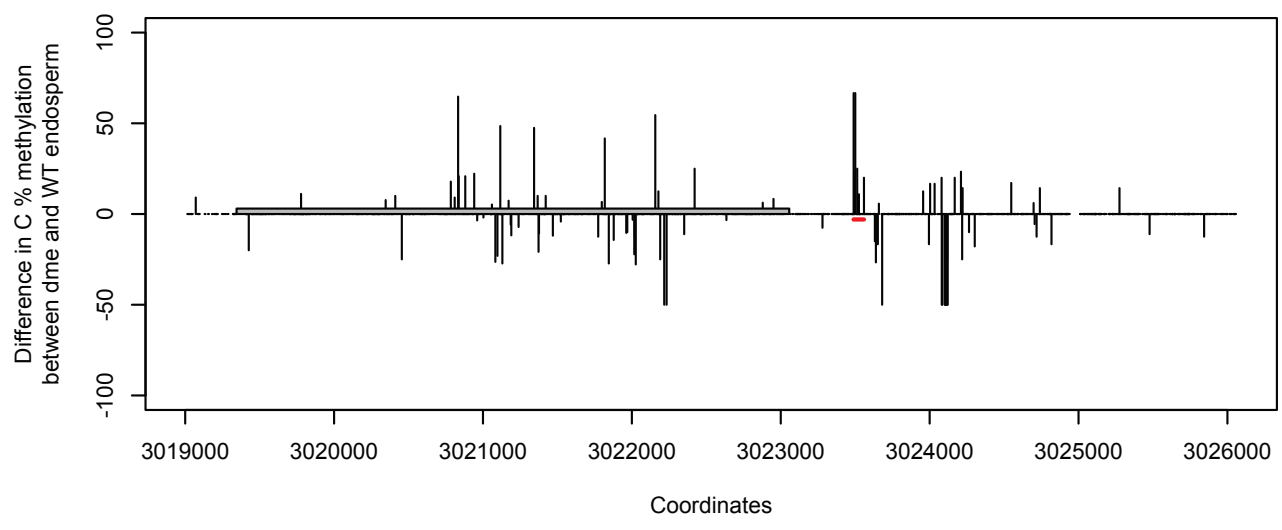

B

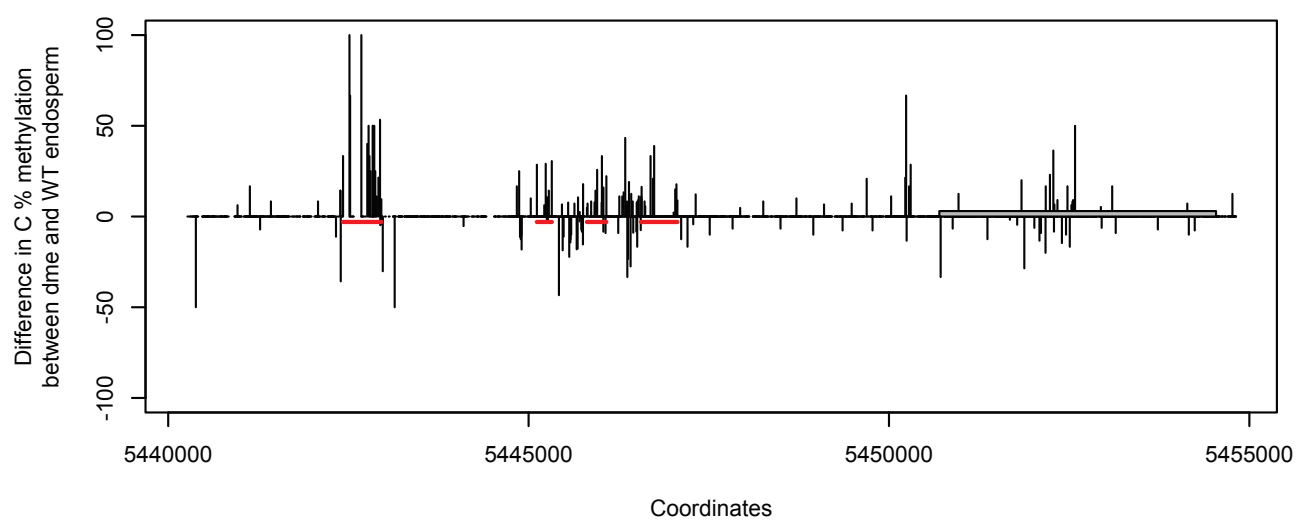

C

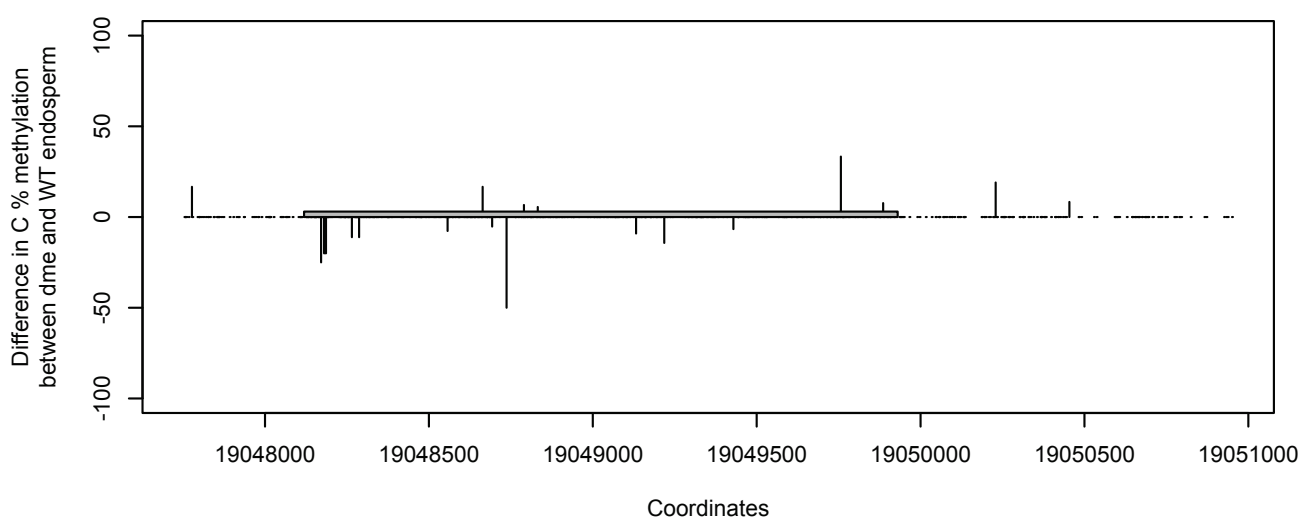

Figure 4 DMRs are located in the vicinity of the ATCDC48 and PDE120 loci. Difference in methylation percentage between dme and wildtype endosperm for cytosines in the vicinity of the ATCDC48 (A), PDE120 (B) and MS5-like (C) loci. Grey boxes represents the gene body (5'-3' orientation), red bars highlight DMRs with potential to act as ICRs. 
suggest that the imprinted MS5-like gene is less likely to be regulated via a methylation-dependent mechanism than the imprinted genes ATCDC48 and PDE120.

\section{Expression levels of imprinted genes ATCDC48 and PDE120 are regulated by methylation pathways} In order to confirm whether DNA methylation changes are associated with altered expression levels of our novel imprinted genes, we performed qRT-PCR on cDNA derived from seedlings of met1-3 plants and found that there is a significant aberrant induction of the imprinted MEGs ATCDC48A and PDE120 in met1-3 mutants (Figure 5A). In concordance with the failure to detect a candidate DMR for MS5-like, no such induction occurred for this gene (Figure 5A). Interestingly, however, seeds generated by fertilising wild-type Arabidopsis thaliana with pollen from met13 plants did not cause a reactivation of the paternal allele of any of the three genes (Figure 5B). The maternal FIS-complex has also been recently shown to regulate imprinting of certain MEGs [37,44-46]. However, for the three imprinted loci of focus in this study, we found that fertilising fis 2 plants with wild-type pollen did not lead to any loss of imprinting either (Figure
5B). Overall, this could imply that the proximal DMRs we have identified do not function as ICRs for these imprinted loci. Alternatively, it may suggest the existence of a subset of imprinted MEGs in which imprinted status and expression levels are regulated via a MET1 - and DME/FIS-independent pathway. The lack of response of these three genes to these epigenetic modifier pathways offers a further explanation for the failure of Hsieh et al. [24] to detect ATCDC48A, MS5like and PDE120 as imprinted MEGs, as their filtering approach compared numbers of sequence reads in wild-type crosses with those crossed to such epigenetic modifier backgrounds.

\section{Discussion}

In comparison with current knowledge of genomic imprinting (i.e. regarding number of imprinted genes and regulatory mechanisms) in mammalian genomes, the study of genomic imprinting in plants has been hindered by the low number of imprinted genes that have been reported and studied to date. In this study, we have sought to address this by identifying novel imprinted genes in the model plant Arabidopsis thaliana and considering our results in the light of screens

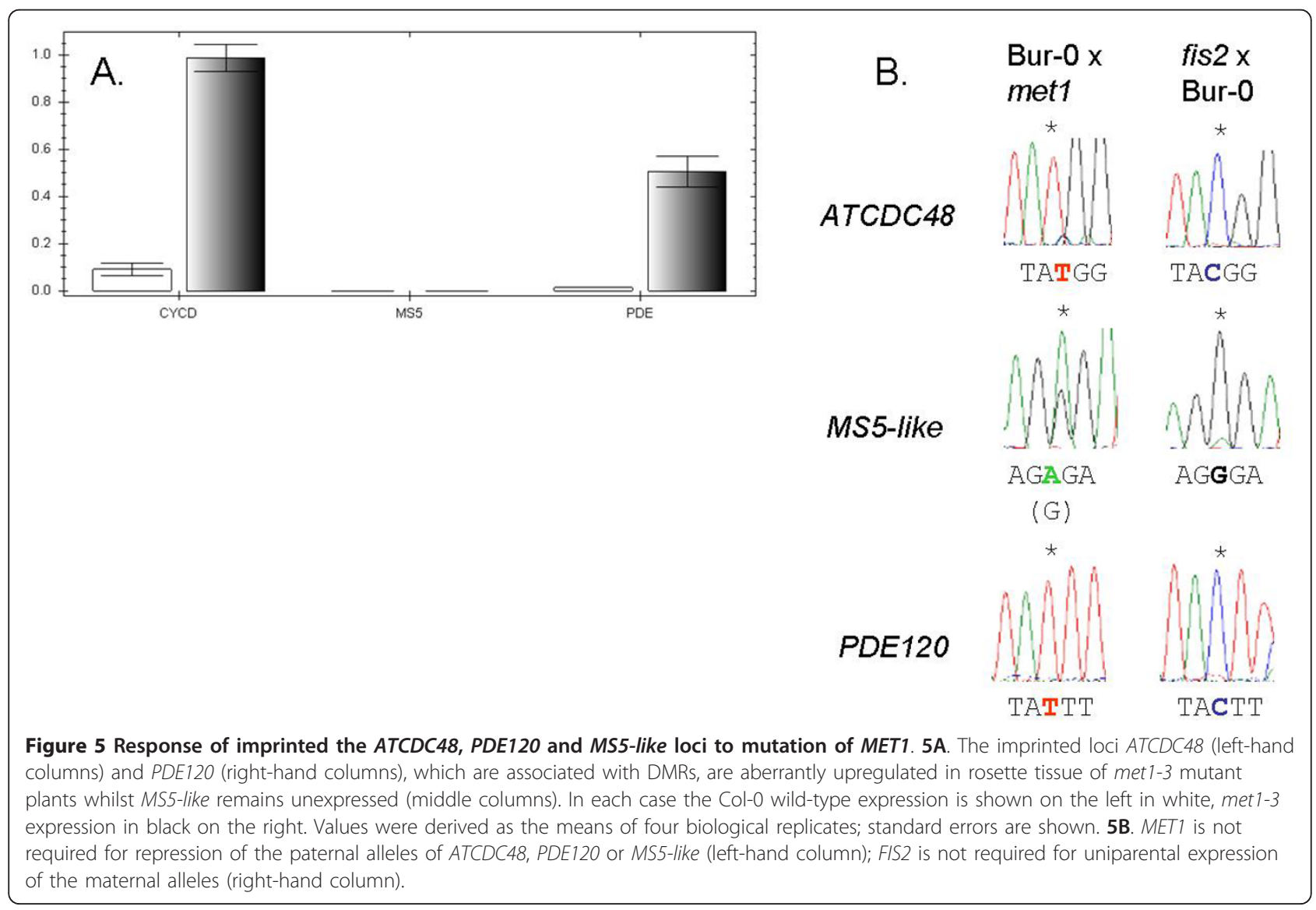


performed by others, and of current theories concerning the regulation of imprinting in plants.

In this study, we have conducted a genome-wide allele-specific expression analysis screen using cDNAAFLP to identify 93 maternally expressed TDFs from a total of 4500 polymorphic allele-specific TDFs. Some of these may represent candidate maternally expressed genes regulated by imprinting in the model plant Arabidopsis thaliana. To identify the genes represented by each TDF, we developed a novel bioinformatics software program called GenFrag which can directly identify genes (in well annotated sequenced genomes e.g. Col-0 accession) based only on the size of the TDF and the selective nucleotides of the primers used to generate the TDF. Although cDNA-AFLP is an early generation transcriptomics platform, as a technique it has some distinct advantages over probe hybridisation based approaches such as microarrays. These advantages include: (a) applicability to any species (including species with no genomic information), (b) low cost and reproducibility, (c) small amounts of RNA template needed, (d) detection of lowly expressed genes and (e) high specificity to distinguish closely related genes [47-50]. However, one of the most time-consuming steps in the cDNA-AFLP technique is the excision of TDFs from gels so that the TDF can be sequenced (typically following amplification and/or subcloning into a plasmid). To increase the throughput of gene identification in cDNA-AFLP experiments involving species with sequenced and well annotated genomes (such as Arabidopsis thaliana), we developed the GenFrag bioinformatics software program.

There have been previous efforts to develop bioinformatic approaches to improve the efficiency of (cDNA-) AFLP techniques. The large amount of DNA sequence data available for several species has been used for in silico predictions of virtual transcript profiles. Tailormade software, such as AFLPinSilico [51] and GenEST $[52,53]$, allow high-throughput identification of AFLP and cDNA-AFLP TDFs for Arabidopsis thaliana and Globodera rostochiensis, respectively. These in silico approaches were also developed to enable experiment simulations, decreasing the time needed for AFLP optimisation, and the number of samples which need to be processed [51-53]. The GenFrag program developed in this study is designed to facilitate high throughput direct identification of genes from cDNA-AFLP experiments with fully sequenced well-annotated genomes such as that of Arabidopsis thaliana. We have made the GenFrag program freely available to the research community at: http://www.nem.wur.nl/UK/Research/bio/.

In our study to identify novel imprinted genes in Arabidopsis thaliana, we applied the GenFrag program to the 93 TDFs displaying a maternal-specific expression pattern, and could thereby identify 52 maternally expressed genes (MEGs) in Arabidopsis thaliana (Table 1). By filtering for expression within seeds and enrichment within endosperm tissues, we ranked 18 MEGs on the basis of the absolute difference of their expression levels between the seed coat and the endosperm (Table 2). The identification of MS5-like and PDE120 was also supported by alternative approaches i.e. comparison with the dataset of Day et al. ([36]; Table 1) and ranking by ratio of Endosperm/Seed Coat expression (Additional file 6 Table S5). For any given gene expressed in the developing seed, it is difficult to separate both the absolute and relative contributions of the different seed tissues, especially given their differing ploidies (triploid in the endosperm, diploid maternal in the seed coat, diploid hybrid in the embryo) and the differences in cellular/nuclear abundance for the different tissues (seed coat, endosperm, embryo). As the contributions to total transcription are normalised against units of RNA no direct determination of the absolute contributions from each seed tissue is possible. However, we can demonstrate that biallelic expression in the seed is detectable at the developmental stage we sample through use of a biallelic endosperm expressed gene (PHE2) as a positive control (Table 3). Our approach does have the advantage of allowing a focus on highly expressed genes, whose transcripts in seeds 4 dap are least likely to have been maternally deposited in the central cell prior to fertilisation. The endosperm is transcriptionally active immediately following fertilization, such that maternally deposited, long-lived RNAs are unlikely to play an important role [34] or be found at high levels in endosperm tissues 4 dap. This contrasts with the early development of the embryo, where expression in the embryo is maternallybiased ( $88 \%$ of transcripts at the $2-4$ cell stage, for example), with paternal alleles subsequently becoming reactivated at the later globular stages of embryo development [32]. Hence, the top ranked endospermenriched genes identified in our study can be considered to be the most likely imprinted genes (Table 2).

A striking finding in our study is that there is little overlap in terms of genes detected between all of the different screens for imprinted genes in Arabidopsis thaliana conducted to date, including our study (Additional file 10 Figure S5). Possible explanations for such lack of overlap can include (a) use of different accessions (genetic backgrounds); (b) use of samples from different developmental stages (where the relative abundance and contribution of embryo versus endosperm tissues will differ); (c) use of different filtering criteria; (d) use of different experimental approaches for isolation of seed, embryo and endosperm tissues and RNA from each tissue; and (e) use of different transcriptome profiling platforms and bioinformatic pipelines. In 
this study we demonstrate that the imprinted genes we have identified are unlikely to be detected at the later developmental stage used by Hsieh et al. [24], whilst the lack of overlap between the next-generation sequencing approaches of Hsieh et al. (2011) and Wolff et al. [23] is likely contributed to the analysis of different time points (7-8 DAP versus 4 DAP) and different accessions (Col-0 $\times$ Ler-0 versus Col-0 $\times$ Bur- 0 ). There is some overlap (7 genes) between the RNA sequencing approach of [23] (Col-0 $\times$ Bur-0 crosses) and a screen for genes regulated by DMRs in Col-gl X Ler-0 crosses [25] suggesting that DMRs may control gene-specific imprinting for a limited number of loci, and/or that their ability to do so may vary according to different genetic backgrounds. Although it seems likely that all these approaches have identified imprinted genes it would seem that detection of imprinted loci (gene-specific or allele-specific) may be dependent upon accessions (genetic backgrounds), developmental stages sampled and experimental methodology. These factors may introduce significant variation between the results of different studies. Given the increasing numbers of allele-specific expression effects being detected in plants, it may be opportune for the imprinting research community to develop some common standards for the definition and validation of imprinted genes in flowering plants (see also [11]).

For the top three ranked genes ATCDC48, PDE120 and MS5-like, using LCM, we could independently detect expression of these genes in 4 dap seed tissues (seed coat, endosperm and embryo) (Additional file 7 Figure S2). For ATCDC48 and PDE120 we also confirmed that expression was low in pre-fertilized ovules but increased during the course of seed development (Figure 1A, B), which is consistent with these genes being subject to post-fertilisation expression in the developing seed (i.e. not maternally deposited). We confirmed that all three of these endosperm-expressed genes are maternally expressed in 4 dap reciprocal F1 hybrid seeds from different accessions and hence represent novel cases of gene-specific imprinting in Arabidopsis thaliana (Figures 2 and 3). While ATCDC48 and PDE120 are subject to binary imprinted expression, MS5-like shows a preferential maternal expression pattern of imprinting [9,21], as some paternal expression is also detected (Figure 2). Although the expression levels of MS5-like were similar in Col-0 and Ler-0 (Figure 1), and in the pattern determined for Ws-0 (Seed Genes Network), the extent of imprinting did vary, with the C24 and Bur-0 alleles displaying a greater extent of imprinting when paternally inherited.

ICRs of imprinted genes often overlap with DMRs. Hence, we considered that our top-ranked imprinted genes ATCDC48, PDE120 and MS5-like might contain candidate DMRs in their genomic vicinity and that, if so, these could be candidate ICRs. We could identify DMRs upstream of PDE120 and one DMR downstream of $A T C D C 48$ that could potentially act as ICRs (Figures $4 \mathrm{~A}$ and $4 \mathrm{~B}$ ). However, the difference in methylation between wild-type and $d m e$ endosperm did not reveal any DMR for MS5-like (Figure 4C). Expression of $D M E$ in the central cell leads to hypomethylation of the maternal genome. However, the methylation data used [43] represent the global methylation status of both the maternal and paternal genomes of the endosperm. This could explain why no DMR could be identified for MS5like. Control of imprinting at the MS5-like locus may be independent of DNA methylation, or be regulated by a DMR far distal to the gene. Methylation-independent imprinting has been observed for some imprinted loci in mammals [54] and histone methylation by Polycomb group proteins has been shown to regulate several imprinted genes in plants $[37,44,55]$. Our results indicate that lack of MET1 in the male gamete has no effect on imprinting of ATCDC48, PDE120 and MS5-like in the developing seed. In contrast, we find that lack of $M E T 1$ leads to overexpression of ATCDC48 and $P D E 120$ in vegetative leaf tissues. No effects of lack of MET1 in vegetative tissues were observed for MS5-like. Taking into consideration the recent findings of [23] and previous reports showing that PcG complexes regulate imprinting [37,44-46], we also tested for possible effects of the maternal FIS-complex on regulation of the three maternally expressed imprinted genes and found that fertilising fis 2 plants with wild-type pollen did not lead to any loss of imprinting. Hence, alternative epigenetic pathways are likely to regulate imprinting of MS5like. Such regulation can neither be ruled out for ATCDC48 and PDE120. Further characterization of the imprinted ATCDC48, PDE120 and MS5-like loci will provide opportunities for increasing our understanding of the epigenetic mechanisms involved in the regulation of genomic imprinting in angiosperms.

The maternally expressed imprinted gene, $A T C D C 48 A$, is a homohexameric AAA(+) ATPase chaperone implicated in cell cycle control and cell proliferation. CDC48/p97 represents a highly conserved protein which plays a role as an initiation factor for DNA replication in many species [56] and has been shown to be essential in a wide range of multicellular and unicellular organisms [57]. In plants, the CDC48A protein has been shown to physically interact with the SOMATIC EMBRYOGENESIS RECEPTOR LIKE KINASE 1 (SERK1) protein [58,59]. The Arabidopsis thaliana genome contains three CDC48 loci, ATCDC48A (At3g09840), ATCDC48B (At3g53230) and ATCDC48C (At5g03340). ATCDC48A can functionally complement CDC48 mutants of Saccharomyces cerevisiae [56], and loss of the PUX1 negative regulator of ATCDC48 leads 
to accelerated plant growth due to increased cell division and expansion [60]. Additional studies in Arabidopsis thaliana conducted with T-DNA knockout lines of AtCDC48A have demonstrated that homozygous null seedlings are viable until 5 days old but die shortly thereafter. It was also demonstrated that null Atcdc48a alleles have a drastically reduced transmission efficiency through the male gametophyte (i.e. ATCDC48A is essential for normal pollen germination and tube elongation) [57].

Our results indicate that $A T C D C 48 A$ is maternally expressed and subject to genomic imprinting in the developing seed (endosperm) (Figures 1, 2 and 3). Although the imprinting status of the maize homolog of $A T C D C 48 A$ has not yet been determined, it is possible that imprinting of the maize homolog of ATCDC48A (or other cell-cycle genes) could be responsible for the dosage effects on cell-cycle progression observed in endosperm from interploidy crosses of maize [61]. While a clear role for ATCDC48 in the control of DNA replication in plant cells has not yet been established, our findings that ATCDC48 is a maternally expressed imprinted gene in developing endosperm resonates with a role in controlling proliferation as suggested for imprinted genes by the parental conflict theory [12].

Less is known from a functional perspective regarding the other two imprinted genes identified in this study. The MS5-like maternally expressed imprinted gene has sequence similarity to Male Sterile 5 (MS5), a gene that has been shown to be essential for male meiosis in Arabidopsis thaliana [62]. MS5-like also displays sequence similarity with the sulphur deficiency-induced gene AtSDI1 [63].

The maternally expressed imprinted gene PDE120 is annotated as a pigment defective embryo (pde) mutant in the SeedGenes database $[64,65]$. The nuclear encoded PDE120 locus encodes the TIC40 protein which is a component of the protein import apparatus of the inner envelope of the chloroplast [66]. The identification of a maternally expressed imprinted nuclear gene which encodes a protein product targeted to the maternallyinherited chloroplasts could be suggestive of selection for imprinting at nuclear loci where strong control by maternally-inherited alleles of chloroplast function is essential [67].

\section{Conclusions}

In this study we have identified 52 maternally expressed genes in siliques containing reciprocal F1 hybrid seeds. We have developed and employed a novel bioinformatics tool called GenFrag to facilitate higher-throughput analysis of cDNA-AFLP experiments on organisms with well-annotated sequenced genomes. We ranked the 52 maternally expressed genes according to their relative expression levels in the endosperm versus seed coat tissues at the globular embryo stage and chose the three top-ranked imprinted candidate genes for further investigation. We confirmed expression of the three candidates in 4 dap seeds by LCM RT-PCR and further confirmed maternal-specific expression of the three genes in 4 dap F1 hybrid seeds generated with different Arabidopsis thaliana accessions. Taken together, our results indicate that $A T C D C 48$ is a maternally expressed imprinted gene in the developing Arabidopsis thaliana seed, and is likely imprinted in the endosperm and perhaps the embryo. Confirmation of imprinted maternal expression was also demonstrated for the other two topranked genes PDE120 and MS5-like. Where present, DMRs for each of the three imprinted genes and the 18 maternally expressed genes in Table 2 were identified and posited as putative ICRs. However, analysis of the imprinted ATCDC48, PDE120 and MS5-like loci with the candidate modifiers met1-3 and fis 2 indicates that the regulation of imprinting at these three genes is independent of DNA methylation and the FIS-complex. Overall, our study identifies novel maternally expressed genes in Arabidopsis thaliana seed and validates three genes (ATCDC48, PDE120 and MS5-like) as novel maternally expressed imprinted genes in Arabidopsis thaliana seed. Further analysis of the genes identified here and by others will accelerate efforts to increase our understanding of the epigenetic regulatory mechanisms and evolution of imprinted genes in flowering plants.

\section{Methods}

\section{Plant growth and generation of cDNA}

Arabidopsis thaliana L. of accessions Col-0, Ler-0, C24 and Bur-0 were grown on 8 parts Westland multipurpose compost (Dungannon, N. Ireland): 1 part perlite: 1 part vermiculite under the following conditions: 200 $\mu \mathrm{mol} \mathrm{m} \mathrm{s}^{-2}$ at $21^{\circ} \mathrm{C} / 18^{\circ} \mathrm{C}$ and a $16: 8 \mathrm{hr}$ light:dark cycle. F1 hybrid seeds were generated via reciprocal crosses of Col-0 and Ler-0, Bur-0 and C24 accessions [24,25]. Plants were manually emasculated before anthesis and reciprocally crossed by hand under a Leica MZ6 dissecting microscope (Leica Microsystems CMS GmbH, Ernst-Leitz-Straße 17-37, Wetzlar, D-35578, Germany) using Dumostar No. 5 tweezers (Dumont Biology, Switzerland). Siliques and seeds were harvested at the time points described. mRNA was extracted in combination with on-column DNase treatment using an RNase-free DNase kit (Qiagen, USA). $5 \mu \mathrm{g}$ of total RNA were hybridized to biotinylated oligo dT which binds the streptavidin-coated PCR tube wall (mRNA Capture Kit, Roche) and cDNA synthesis performed (Quantitect Reverse Transcriptase kit, Qiagen). Quality control was performed on the Agilent 2100 Bioanalyzer (Agilent Technologies Schweiz AG, Basel, Switzerland). Samples were stored at $-80^{\circ} \mathrm{C}$ prior to use. 


\section{CDNA-AFLP}

cDNA from siliques was generated as described, digested with restriction enzymes BstYI and MseI and ligated with adapters complementary to the restriction site of BstYI (5'-CTCGTAGACTGCGTAGTGATCYGATCCGTTCA-3 and 3'-CATCTGACGCATCACTAGRCTAGGCAAGT-5) and MseI (5'GACGATGAGTCCTGAGTAACACTGGATCATG-3' and 3'-CTACTCAGGACTCATTGTGAGGTAGTAC-5). The ligated fragments were selectively amplified a first time using $M s e$ I primer (5'-GATGAGTCCTGAGTAA$3^{\prime}$ ) and BstYI primers (5'-GACTGCGTAGTGATCCN-3 and 5'-GACTGCGTAGTGATCTN-3'). The amplified fragments were diluted 1:20 and amplified a second time using 128 primer combinations ( 8 Bst YI possible primers 5'-GACTGCGTAGTGATCCNN-3 and 5'GACTGCGTAGTGATCTNN-3' $\times 16 \mathrm{MseI}$ possible primers 5'-GATGAGTCCTGAGTAANN-3' = 128 combinations). Products were run on polyacrylamide gels and visualised with the GelDoc-ItTM Imaging System (Ultra-Violet Products Ltd., Cambridge, UK). Samples were processed using the 16-capillary $3130 \times 1$ Genetic Analyser (Applied Biosystems Inc.). $0.5 \mu \mathrm{l}$ reaction products were mixed with $0.4 \mu \mathrm{l}$ Internal Lane Standard 600 ROXTM size standard (Promega, WI, USA) or GeneScanTM 500 ROXTM size standard (Applied Biosystems, UK), in $9 \mu \mathrm{l} \mathrm{Hi-Di} \mathrm{Formamide} \mathrm{(Applied}$ Biosystems, UK). Fragments were analysed in a multiplex run and visualised with $B s t \mathrm{YI}+\mathrm{C}$ and $B s t \mathrm{YI}+\mathrm{T}$ primers, respectively labelled with the fluorescent dyes JOE and 6-FAM. Samples were analysed using the GeneMapper v3.7 software, which assigned each TDF an allelic label, or bin, based on its size as determined by comparison to the ILS600-C marker (Promega). Bin assignment permitted a variation of $\pm 0.5 \mathrm{bp}$ in the determined size. For cDNA-AFLP samples generated with a given primer combination, the two parental lines, Col- $0 \times$ Col- 0 and Ler- $0 \times$ Ler- 0 , and the two reciprocal hybrids, Col- $0 \times$ Ler- 0 and Ler- $0 \times$ Col- 0 were analysed together within a run to allow identification of polymorphic and differentially expressed TDFs. Fragment-sizing and allele-calling parameters for GeneMapper were normalized to the data using the default Sum-of-Signal method; alleles common between samples were not deleted. This generated electropherograms matching detected peaks with their allele calls, from which genotypes were derived.

\section{Development of GenFrag program \& software}

We downloaded the two datasets containing the available full-length Arabidopsis thaliana cDNAs from the TIGR v.4.0 (released March 2005) and TAIR v.7 databases at ftp://ftp.tigr.org/pub/data/a_thaliana/ath1/ SEQUENCES/ and ftp://ftp.arabidopsis.org/home/tair/ Sequences/ (released April 2007) respectively.
Arabidopsis thaliana ESTs were downloaded fromhttp:// www.plantgdb.org/download/Download/Sequence/ESTcontig/Arabidopsis_thaliana/current_version/Arabidopsis_thaliana.mRNA.PUT.fasta.bz2 and a dataset of alternative splicing variants from TIGR-Atg database (release June 2003) at http://www.tigr.org/tdb/e2k1/ ath1/altsplicing/splicing_variations.shtml.

GenFrag expands on the earlier GenEst package [53] by providing a web interface which is publicly available at the URL: http://www.nem.wur.nl/UK/Research/bio/. GenFrag provides full named support for all known restriction enzymes as listed in REBASE [68], additional support for primer combinations, their size corrections, and a listing of mismatched fragment sizes. GenFrag also allows a subset of experimental allelic fragments to be selected for analysis on the basis of the potential interest of genes in a candidate sequence list i.e. rather than sequencing all fragments. The GenFrag software is written in Ruby, and can be run on all platforms supported by Ruby, including Windows, OSX, Linux and the Java virtual machine. The restriction enzyme module is available as part of the Open Bioinformatics Foundation BioRuby toolkit [69] and includes all known restriction enzymes by name. Genomic information can be read in any BioRuby supported format, including FASTA. The web interface is written in Ruby on Rails, and SQLite is used for caching searches. GenFrag software can be used in two ways: through a public web interface and as a software module in a computing pipeline.

\section{Expression analysis}

Microarray data of gene expression levels and absence calls from Seedgenenetwork (Harada-Goldberg Arabidopsis Laser Capture Microdissection Gene Chip Data Set, http:// seedgenenetwork.net) were downloaded from Gene Expression Omnibus [70], accession numbers GSM284397 and GSM284398 (seed coat), GSM284390 and GSM284391 (peripheral endosperm), GSM284388 and GSM284389 (micropylar endosperm), GSM284392, GSM284393 and GSM284394 (chalazal endosperm) and GSM284384 and GSM284385 (embryo). The developmental stage sampled by these experiments is the globular stage of embryo development. The mean expression value of all replicates was used. The following genes did not have probes: At1g12420, At1g55320, At2g45315, At3g21465, At4g01000, At4g25315, At5g04895, At5g35737 and At5g40240. Probes for At4g37530 and At1g14880 also matched another gene so were omitted from the analysis due to the possibility of ambiguous results.

\section{LCM}

Siliques of emasculated and hand-crossed plants of accession Ler-0 were collected and directly transferred 
to an ASP200 embedding machine (Leica Microsystems $\mathrm{GmbH}$, Wetzlar, Germany) and dehydrated at room temperature in a graded ethanol series (1 hour at $70 \%, 3$ $\times 1$ hour at $90 \%, 3 \times 1$ hour at $99.98 \%)$ and in xylol (2 $\times 1$ hour and $1 \times 75$ minutes) which was substituted by Paraplast X-tra embedding media (Roth AG, Arlesheim, Switzerland $)$ at $56^{\circ} \mathrm{C}(2 \times 1$ hour, $1 \times 3$ hours $)$, poured into paraffin blocks and stored at $4^{\circ} \mathrm{C}$. Paraffin blocks were cut to $10 \mu \mathrm{m}$ thin sections on an RM2145 microtome (Leica Microsystems GmbH, Wetzlar, Germany) and mounted on nuclease-free membranes held in metal frame slides in methanol, dried overnight at $42^{\circ} \mathrm{C}$ and deparaffinised in xylol at $56^{\circ} \mathrm{C}(3 \times 10$ minutes $)$. Microdissection was performed on thin sections of siliques using the MMI CellCut Plus laser capture microscope (MMI Molecular Machines and Industries AG, Glattburg, Switzerland) to generate circa 150 cuts (1500 cells) per sample. Total RNA was extracted from pooled samples using the PicoPure RNA isolation kit (Arcturus Engineering, Mountain View, CA 94043-4019, USA) and single-stranded cDNA generated using the NuGEN WT-Ovation Pico RNA Amplification System (NuGEN Technologies Inc., Brockville, Canada).

\section{RT-PCR}

Primers for the three top ranked candidate genes were designed using the Universal ProbeLibrary Assay Design Center (Roche, Switzerland, http://www.roche-appliedscience.com) Identical PCR conditions were used for all genes, with $\mathrm{Tm}$ of $59^{\circ} \mathrm{C}$ and 40 amplification cycles. Two replicates were performed (data not shown), one representative result is shown for the three top ranked candidate imprinted genes analysed (Additional file 7 Figure S2). Quantitative RT-PCR was performed on biological triplicate samples using SYBR Green master mix (ABI) and run on a C1000 Thermal CycLer incorporating the CFX Real-Time System. Details of all primers are available on request.

\section{DNA sequencing \& QUASEP}

Exonic SNPs between Arabidopsis thaliana accessions were identified at The Arabidopsis Information Resource [71] (PERL0437780 for ATCDC48, PERL0895299 for PDE120, PERL0626585 for MS5-like and Exon 2, $2345566(\mathrm{C} / \mathrm{T})$ for PHE1). cDNA from seeds of reciprocal Col- $0 \times \mathrm{C} 24$ and Col- $0 \times$ Ler- 0 crosses was generated as described. Sequences surrounding the SNPs were amplified by PCR performed under standard conditions with GoTaq (Invitrogen) and sequenced by GATC. Quantification of maternally- and paternally-derived SNPs was performed via QUASEP (Quantification of Allele-Specific Expression by Pyrosequencing). RT-PCR was performed with Quantitect RT kits according to manufacturer's instructions. PCR was performed on cDNA using one biotinylated primer per pair using sequences adapted from assays designed by PSQ assay software (sequences available on request). Mean values of parental expression were calculated from at least three replicates. Genomic DNA and the genes FWA, PHE1 and PHE2 were used as controls.

\section{Identification of DMRs}

High-throughput bisulfite sequencing data of Arabidopsis thaliana wild-type endosperm and endosperm from seeds deficient for a maternal DME allele [43] were retrieved from ArrayExpress (http://www.ebi.ac.uk/ arrayexpress, accession number E-GEOD-15922), corresponding to the TAIR 8 version of the genome. The percentage of methylation at cytosines situated between the genes immediately upstream and downstream of our candidates was calculated. Regions that showed a difference between $d m e$ and wild-type endosperm cytosine methylation percentages were identified as DMRs and potential ICRs.

\section{Additional material}

Additional file 1: Table S1 - Known imprinted genes in flowering plants. In the angiosperms, eleven imprinted genes had been reported from Arabidopsis thaliana and related species, six from maize and one from rice. All but three are expressed solely from the maternally inherited allele. With the exception of MEDEA, for which conflicting reports have been published (for discussion see [72]), all imprinted Arabidopsis thaliana genes show mono-allelic expression only in the terminally differentiating endosperm, whereas maize Meel clearly shows imprinted expression in the maize embryo.

Additional file 2: Figure S1 - Flow chart of the GenFrag program Generation of the Transcript Derived Fragment (TDF) by poly-A capture of mRNA from silique tissue, followed by two digestions using first BstYl then $\mathrm{Msel}$ restriction enzymes. The fragments were ligated to adapters and selectively amplified using the primer combinations described (Additional file 4, Table S3). Transcript sizes were then determined on the ABI3130xl Genetic Analyzer. TDFs which were called as present in the maternal hybrid cross direction but absence from the reciprocal cross were considered as being derived from candidate MEGs (Additional file 3, Table S2). Subsequently, transcript sequences were assigned to progenitor genes by GenFrag using publicly available Arabidopsis transcript sequences. The transcript goes through two in silico digestions using first BstYl then Msel restriction enzymes to produce one fragment per transcript mimicking the in vitro protocol from which the gene identify is determined (Table 1).

Additional file 3: Table S2 - Relative proportions of uniparental TDFs expressed in siliques as determined by cDNA-AFLP of hybrid Col- $0 \times$ Ler- 0 reciprocal crosses across three timepoints.

Additional file 4: Table S3 - Identification of 93 TDFs showing maternal-specific expression using the GenFrag software. 93 TDFs showing maternal-specific expression were analysed by GenFrag. Gene identitites were predicted using combinations of primers designed against BstYY and Msel cutting sites ( $3^{\text {rd }}$ and $4^{\text {th }}$ columns) and the size of the TDF as determined by capillary electrophoresis ( $5^{\text {th }}$ column) as unique identifiers. Single unique genes were predicted for 52 TDFs out of the 93 maternal-specific TDFs (7 $7^{\text {th }}$ column, TDFs 1-52). A further 21 TDFs (TDFs 53-73) rendered non-unique predictions and 20 others (TDFs 74-93) could not be matched by GenFrag to any known Arabidopsis thaliana sequence. For 8 TDFs, the predicted size (marked with *) 
differed from that determined by capillary electrophoresis, by amounts indicated in parentheses.

Additional file 5: Table S4 - Lack of detection of known imprinted genes is due to lack of SNPs in restriction sites. Msel cuts in the T/ TAA context, Bst YI cuts in the R/GATCY context (source: SALK SNP viewer, TAIR)

Additional file 6: Table S5 - Relative expression levels of maternallyexpressed seed genes in the endosperm and seed coat. Genes detected as maternal by CDNA-AFLP with a log2-ratio of higher than 1 (indicating expression twice as high in endosperm vs. seed coat) are listed.

Additional file 7: Figure S2 - Analysis of expression profiles of ATCDC48, PDE120 and MS5-like in LCM tissues of Arabidopsis thaliana seeds (4 dap). Results of RT-PCR performed on CDNA derived from LCM endosperm (ES), seed coat (SC) and embryo (EM) tissues, shown for one representative replicate of two. ACT11 and UBC9 were used as loading controls.

Additional file 8: Figure S3 - Confirmation of maternal expression of CDNA-AFLP candidate genes in crosses to Ler- 0 . CDNA from 4 dap tissue was amplified and sequenced from F1 Ler- $0 \times$ Col-0 hybrid seeds and MS5-like and PDE120 found to be maternally expressed.

Additional file 9: Figure S4 - Confirmation of six further CDNA-AFLP genes as maternal in seed tissue. CDNA from F1 4 dap seed tissue was amplified and sequenced. In each case, CDNA from Col-0 $\times$ C24 is shown on the left, C24 $\times$ Col-0 is shown on the right. SNPs are marked with asterisks.

Additional file 10: Figure S5 - Divergent candidate imprinted genes identified by different screens in Arabidopsis. The overlap between maternally-expressed genes identified from this CDNA-AFLP screen performed in 3-5 dap Col-0 $\times$ Ler-0 crosses (red); those predicted from analysis of DMRs identified from Col-0 $\times$ Ler-0 endosperm (blue, [25]) and those identified by next generation screening approaches ([24] yellow; [23]; green) (see descriptions in Introduction).

Additional file 11: Figure S5 - Identification of DMRs located in the vicinity of candidate imprinted genes (Table 2) and other maternally inherited genes. Difference in methylation percentage between dme and wild-type endosperm for cytosine bases in the vicinity of three candidate imprinted genes (Table 2). Grey boxes represent the gene body in a $5^{\prime}-3^{\prime}$ orientation, red bars highlight DMRs.

\section{Acknowledgements}

This work was funded through grant funding to CS from the Irish Department of Agriculture, Fisheries and Food (grant RSF 07-534) and Science Foundation Ireland (SFI) (grants 02/IN.1/B49 and 08/IN.1/B1931) Funding support to UG is also acknowledged from the the 'Stiftung für wissenschaftliche Forschung' of the University of Zürich. The support of COST Action FA0903 (HAPRECI) on "Harnessing Plant Reproduction for Crop Improvement" is acknowledged. The authors thank the anonymous reviewers for their comments and suggestions.

\section{Author details}

${ }^{1}$ Genetics and Biotechnology Lab, Botany and Plant Science, National University of Ireland Galway (NUIG), C306 Aras de Brun, University Road, Galway, Ireland. '2Laboratory of Nematology, Wageningen University, Droevendaalsesteeg 1, Wageningen, The Netherlands. ${ }^{3}$ Department of Biology and Zürich-Basel Plant Science Center, Swiss Federal Institute of Technology, ETH Centre, CH-8092 Zürich, Switzerland. ${ }^{4}$ Department of Plant Biology and Forest Genetics, Uppsala BioCenter, Swedish University of Agricultural Sciences, SE-75007 Uppsala, Sweden. ${ }^{5}$ Institute of Plant Biology and Zürich-Basel Plant Science Center, University of Zürich, Zollikerstrasse 107, CH-8008 Zürich, Switzerland. ${ }^{6}$ Silicon Life Sciences, Minneapolis, MN, USA

\section{Authors' contributions}

PMcK designed assays, performed sequencing and pyrosequencing, and prepared the manuscript. SLD performed the CDNA-AFLP screen, analyzed
TDF data, and performed pyrosequencing controls. PP contributed in-silico CDNA-AFLP and, with TJW, developed the GenFrag software. PW generated cDNA sequence traces from Col-0 $\times$ Bur- 0 accessions and crosses to mutant modifiers. MS performed LCM and RT-PCR experiments. MTAD analysed and compared data-sets, determined expression ratios of candidate genes, prepared figure S1 and formatted and edited the manuscript. AF and DD performed qRT-PCR. NTL generated hybrid CDNA and conducted sequencing reactions. AC identified differentially methylated regions and edited the manuscript. TJW and GS supervised the development of GenFrag. CK and UG assisted experimental design, UG supervised and funded the performance of LCM and related expression analyses. CS designed experiments, raised financing for their implementation, oversaw the experiments and development of the project, and prepared the final manuscript. All authors read and confirmed the manuscript.

Received: 7 January 2011 Accepted: 12 August 2011 Published: 12 August 2011

\section{References}

1. Walbot $\mathrm{V}$, Evans MMS: Unique features of the plant life cycle and their consequences. Nature Reviews Genetics 2003, 4(5):369-379.

2. Lord EM, Russell SD: The mechanisms of pollination and fertilization in plants. Annual Review of Cell and Developmental Biology 2002, 18:81-105.

3. Dresselhaus T: Cell-cell communication during double fertilization. Current Opinion in Plant Biology 2006, 9(1):41-47.

4. Berger F: Endosperm: the crossroad of seed development. Current Opinion in Plant Biology 2003, 6(1):42-50.

5. Haughn G, Chaudhury A: Genetic analysis of seed coat development in Arabidopsis. Trends Plant Sci 2005, 10(10):472-477.

6. Brukhin V, Curtis MD, Grossniklaus U: The angiosperm female gametophyte: No longer the forgotten generation. Current Science 2005 89(11):1844-1852.

7. Johnston AJ, Meier P, Gheyselinck J, Wuest SE, Federer M, Schlagenhauf E, Becker JD, Grossniklaus U: Genetic subtraction profiling identifies genes essential for Arabidopsis reproduction and reveals interaction between the female gametophyte and the maternal sporophyte. Genome Biology 2007, 8(10).

8. Scott RJ, Spielman M, Bailey J, Dickinson HG: Parent-of-origin effects on seed development in Arabidopsis thaliana. Development 1998, 125(17):3329-3341

9. Dilkes BP, Comai L: A differential dosage hypothesis for parental effects in seed development. Plant Cell 2004, 16:3174-3180

10. Grossniklaus U, Vielle-Calzada JP, Hoeppner MA, Gagliano WB: Maternal control of embryogenesis by MEDEA, a polycomb group gene in Arabidopsis. Science 1998, 280(5362):446-450.

11. Raissig MT, Baroux C, Grossniklaus U: Regulation and Flexibility of Genomic Imprinting during Seed Development. The Plant Cell Online 2011.

12. Haig D, Westoby M: Genomic Imprinting in Endosperm - Its Effect on Seed Development in Crosses between Species, and between Different Ploidies of the Same Species, and Its Implications for the Evolution of Apomixis. Philosophical Transactions of the Royal Society of London Series BBiological Sciences 1991, 333(1266):1-13.

13. Kinoshita $\mathrm{T}$, Ikeda $\mathrm{Y}$, Ishikawa R: Genomic imprinting: A balance between antagonistic roles of parental chromosomes. Seminars in Cell \& Developmental Biology 2008, 19(6):574-579.

14. Garnier O, Laoueille-Duprat S, Spillane C: Genomic imprinting in plants. Epigenetics 2008, 3(1):14-20

15. O'Connell MJ, Loughran NB, Walsh TA, Donoghue MT, Schmid K, Spillane C: A phylogenetic approach to test for evidence of parental conflict or gene duplications associated with protein-encoding imprinted orthologous genes in placental mammals. Mamm Genome 2010, 21(9-10):486-498

16. Morison IM, Ramsay JP, Spencer HG: A census of mammalian imprinting Trends in Genetics 2005, 21(8):457-465.

17. Vielle-Calzada JP, Thomas J, Spillane C, Coluccio A, Hoeppner MA Grossniklaus U: Maintenance of genomic imprinting at the Arabidopsis medea locus requires zygotic DDM1 activity. Genes Dev 1999, 13(22):2971-2982.

18. Kohler C, Hennig L, Spillane C, Pien S, Gruissem W, Grossniklaus U: The Polycomb-group protein MEDEA regulates seed development by 
controlling expression of the MADS-box gene PHERES1. Genes Dev 2003, 17(12):1540-1553.

19. Tiwari S, Schulz R, Ikeda Y, Dytham L, Bravo J, Mathers L, Spielman M, Guzman P, Oakey RJ, Kinoshita T, et al: MATERNALLY EXPRESSED PAB CTERMINAL, a novel imprinted gene in Arabidopsis, encodes the conserved C-terminal domain of polyadenylate binding proteins. Plant Cell 2008, 20(9):2387-2398.

20. Guo M, Rupe MA, Danilevskaya ON, Yang XF, Hut ZH: Genome-wide mRNA profiling reveals heterochronic allelic variation and a new imprinted gene in hybrid maize endosperm. Plant Journal 2003, 36(1):30-44.

21. Stupar RM, Hermanson PJ, Springer NM: Nonadditive expression and parent-of-origin effects identified by microarray and allele-specific expression profiling of maize endosperm. Plant Physiology 2007, 145:411-425.

22. Shirzadi $R$, Andersen ED, Bjerkan KN, Gloeckle BM, Heese M, Ungru A, Winge P, Koncz C, Aalen RB, Schnittger A, et al: Genome-Wide Transcript Profiling of Endosperm without Paternal Contribution Identifies Parentof-Origin-Dependent Regulation of <italic>AGAMOUS-LIKE36</italic>. PLoS Genet 2011, 7(2):e1001303.

23. Wolff P, Weinhofer I, Seguin J, Roszak P, Beisel C, Donoghue MTA, Spillane C, Nordborg M, Rehmsmeier M, Köhler C: High-Resolution Analysis of Parent-of-Origin Allelic Expression in the Arabidopsis Endosperm. PLoS Genet 2011, 7(6):e1002126.

24. Hsieh T-F, Shin J, Uzawa R, Silva P, Cohen S, Bauer MJ, Hashimoto M, Kirkbride RC, Harada JJ, Zilberman D, et al: Regulation of imprinted gene expression in Arabidopsis endosperm. Proceedings of the National Academy of Sciences 2011, 108(5):1755-1762.

25. Gehring M, Bubb KL, Henikoff S: Extensive Demethylation of Repetitive Elements During Seed Development Underlies Gene Imprinting. Science 2009, 324(5933):1447-1451

26. Kinoshita T, Miura A, Choi Y, Kinoshita Y, Cao X, Jacobsen SE, Fischer RL, Kakutani T: One-way control of FWA imprinting in Arabidopsis endosperm by DNA methylation. Science 2004, 303(5657):521-523.

27. Choi Y, Gehring M, Johnson L, Hannon M, Harada JJ, Goldberg RB, Jacobsen SE, Fischer RL: DEMETER, a DNA glycosylase domain protein, is required for endosperm gene imprinting and seed viability in arabidopsis. Cell 2002, 110(1):33-42.

28. Baroux C, Spillane C, Grossniklaus U: Genomic imprinting during seed development. Homology Effects 2002, 46:165-214.

29. Jullien PE, Berger F: Gamete-specific epigenetic mechanisms shape genomic imprinting. Curr Opin Plant Biol 2009, 12(5):637-642.

30. Villar CB, Erilova A, Makarevich G, Trosch R, Kohler C: Control of PHERES1 imprinting in Arabidopsis by direct tandem repeats. Mol Plant 2009, 2(4):654-660

31. Bachem CWB, vanderHoeven RS, deBruijn SM, Vreugdenhil $D$, Zabeau M, Visser RGF: Visualization of differential gene expression using a novel method of RNA fingerprinting based on AFLP: Analysis of gene expression during potato tuber development. Plant Journal 1996, 9(5):745-753.

32. Autran D, Baroux C, Raissig Michael T, Lenormand T, Wittig M, Grob S, Steimer A, Barann M, Klostermeier Ulrich C, Leblanc O, et al: Maternal Epigenetic Pathways Control Parental Contributions to Arabidopsis Early Embryogenesis. Cell 2011, 145(5):707-719.

33. Haas BJ, Delcher AL, Mount SM, Wortman JR, Smith RK, Hannick LI, Maiti R, Ronning CM, Rusch DB, Town CD, et al: Improving the Arabidopsis genome annotation using maximal transcript alignment assemblies. Nucleic Acids Res 2003, 31(19):5654-5666.

34. Pillot M, Baroux C, Vazquez MA, Autran D, Leblanc O, Vielle-Calzada JP, Grossniklaus U, Grimanelli D: Embryo and endosperm inherit distinct chromatin and transcriptional states from the female gametes in Arabidopsis. Plant Cell 22(2):307-320.

35. Le BH, Cheng C, Bui AQ, Wagmaister JA, Henry KF, Pelletier J, Kwong L, Belmonte $\mathrm{M}$, Kirkbride $\mathrm{R}$, Horvath $\mathrm{S}$, et al: Global analysis of gene activity during Arabidopsis seed development and identification of seed-specific transcription factors. Proceedings of the National Academy of Sciences 2010, 107(18):8063-8070

36. Day RC, Herridge RP, Ambrose BA, Macknight RC: Transcriptome Analysis of Proliferating Arabidopsis Endosperm Reveals Biological Implications for the Control of Syncytial Division, Cytokinin Signaling, and Gene Expression Regulation. Plant Physiology 2008, 148(4):1964-1984.
37. Kohler C, Page DR, Gagliardini V, Grossniklaus U: The Arabidopsis thaliana MEDEA Polycomb group protein controls expression of PHERES1 by parental imprinting. Nat Genet 2005, 37(1):28-30.

38. Tycko B: Allele-specific DNA methylation: beyond imprinting. Hum Mol Genet 2010, 19(R2):R210-220.

39. Meaburn EL, Schalkwyk LC, Mill J: Allele-specific methylation in the human genome Implications for genetic studies of complex disease. Epigenetics 2010, 5(7).

40. Shoemaker R, Deng J, Wang W, Zhang K: Allele-specific methylation is prevalent and is contributed by CpG-SNPs in the human genome. Genome Res 2010, 20(7):883-889.

41. Schalkwyk LC, Meaburn EL, Smith R, Dempster EL, Jeffries AR, Davies MN, Plomin R, Mill J: Allelic skewing of DNA methylation is widespread across the genome. Am J Hum Genet 2010, 86(2):196-212.

42. Kinoshita Y, Saze H, Kinoshita T, Miura A, Soppe WJ, Koornneef M, Kakutani T: Control of FWA gene silencing in Arabidopsis thaliana by SINE-related direct repeats. Plant J 2007, 49(1):38-45.

43. Hsieh TF, Ibarra CA, Silva P, Zemach A, Eshed-Williams L, Fischer RL, Zilberman D: Genome-wide demethylation of Arabidopsis endosperm. Science 2009, 324(5933):1451-1454.

44. Baroux C, Gagliardini V, Page DR, Grossniklaus U: Dynamic regulatory interactions of Polycomb group genes: MEDEA autoregulation is required for imprinted gene expression in Arabidopsis. Genes Dev 2006, 20(9):1081-1086

45. Gehring M, Huh JH, Hsieh T-F, Penterman J, Choi Y, Harada JJ, Goldberg RB, Fischer RL: DEMETER DNA Glycosylase Establishes MEDEA Polycomb Gene Self-Imprinting by Allele-Specific Demethylation. Cell 2006, 124(3):495-506.

46. Jullien PE, Kinoshita T, Ohad N, Berger F: Maintenance of DNA methylation during the Arabidopsis life cycle is essential for parental imprinting. Plant Cell 2006, 18(6):1360-1372.

47. Wenz H, Robertson JM, Menchen S, Oaks F, Demorest DM, Scheibler D, Rosenblum BB, Wike C, Gilbert DA, Efcavitch JW: High-precision genotyping by denaturing capillary electrophoresis. Genome Res 1998 8(1):69-80.

48. Cho RJ, Huang M, Campbell MJ, Dong H, Steinmetz L, Sapinoso L, Hampton G, Elledge SJ, Davis RW, Lockhart DJ: Transcriptional regulation and function during the human cell cycle. Nat Genet 2001, 27(1):48-54.

49. Fukumura R, Takahashi H, Saito T, Tsutsumi Y, Fujimori A, Sato S, Tatsumi K, Araki R, Abe M: A sensitive transcriptome analysis method that can detect unknown transcripts. Nucleic Acids Res 2003, 31(16):e94.

50. Reijans M, Lascaris R, Groeneger AO, Wittenberg A, Wesselink E, van Oeveren J, de Wit E, Boorsma A, Voetdijk B, van der Spek H, et al: Quantitative comparison of CDNA-AFLP, microarrays, and GeneChip expression data in Saccharomyces cerevisiae. Genomics 2003, 82(6):606-618.

51. Rombauts $S$, Van De Peer $Y$, Rouze P: AFLPinSilico, simulating AFLP fingerprints. Bioinformatics 2003, 19(6):776-777.

52. Qin $L$, Prins $P$, Helder J: Linking CDNA-AFLP-based gene expression patterns and ESTs. Methods Mol Biol 2006, 317:123-138.

53. Qin L, Prins P, Jones JT, Popeijus H, Smant G, Bakker J, Helder J: GenEST, a powerful bidirectional link between cDNA sequence data and gene expression profiles generated by CDNA-AFLP. Nucleic Acids Research 2001, 29(7):1616-1622

54. Gribnau J, Hochedlinger K, Hata K, Li E, Jaenisch R: Asynchronous replication timing of imprinted loci is independent of DNA methylation, but consistent with differential subnuclear localization. Genes Dev 2003, 17(6):759-773.

55. Fitz Gerald JN, Hui PS, Berger F: Polycomb group-dependent imprinting of the actin regulator AtFH5 regulates morphogenesis in Arabidopsis thaliana. Development 2009, 136(20):3399-3404.

56. Deichsel A, Mouysset J, Hoppe T: The ubiquitin-selective chaperone CDC48/p97, a new player in DNA replication. Cell Cycle 2009, 8(2):185-190.

57. Park S, Rancour DM, Bednarek SY: In planta analysis of the cell cycledependent localization of AtCDC48A and its critical roles in cell division, expansion, and differentiation. Plant Physiol 2008, 148(1):246-258.

58. Aker J, Borst JW, Karlova R, de Vries S: The Arabidopsis thaliana AAA protein CDC48A interacts in vivo with the somatic embryogenesis receptor-like kinase 1 receptor at the plasma membrane. J Struct Biol 2006, 156(1):62-71. 
59. Aker J, Hesselink R, Engel R, Karlova R, Borst JW, Visser AJ, de Vries SC: In vivo hexamerization and characterization of the Arabidopsis AAA ATPase CDC48A complex using forster resonance energy transferfluorescence lifetime imaging microscopy and fluorescence correlation spectroscopy. Plant Physiol 2007, 145(2):339-350.

60. Rancour DM, Park S, Knight SD, Bednarek SY: Plant UBX domaincontaining protein 1 , PUX1, regulates the oligomeric structure and activity of arabidopsis CDC48. J Biol Chem 2004, 279(52):54264-54274.

61. Jullien PE, Berger F: Parental genome dosage imbalance deregulates imprinting in Arabidopsis. PLoS Genet 2010, 6(3):e1000885.

62. Glover J, Grelon M, Craig S, Chaudhury A, Dennis E: Cloning and characterization of MS5 from Arabidopsis: a gene critical in male meiosis. Plant J 1998, 15(3):345-356.

63. Howarth JR, Parmar S, Barraclough PB, Hawkesford MJ: A sulphur deficiency-induced gene, sdi1, involved in the utilization of stored sulphate pools under sulphur-limiting conditions has potential as a diagnostic indicator of sulphur nutritional status. Plant Biotechnol J 2009, 7(2):200-209.

64. Tzafrir I, Dickerman A, Brazhnik O, Nguyen Q, McElver J, Frye C, Patton D, Meinke D: The Arabidopsis SeedGenes Project. Nucleic Acids Res 2003, 31(1):90-93.

65. Tzafrir I, Pena-Muralla R, Dickerman A, Berg M, Rogers R, Hutchens S, Sweeney TC, McElver J, Aux G, Patton D, et al: Identification of genes required for embryo development in Arabidopsis. Plant Physiol 2004, 135(3):1206-1220.

66. Bedard J, Kubis S, Bimanadham S, Jarvis P: Functional similarity between the chloroplast translocon component, Tic40, and the human cochaperone, Hsp70-interacting protein (Hip). J Biol Chem 2007, 282(29):21404-21414

67. Wolf JB: Cytonuclear interactions can favor the evolution of genomic imprinting. Evolution 2009, 63(5):1364-1371.

68. Roberts RJ, Vincze T, Posfai J, Macelis D: REBASE-restriction enzymes and DNA methyltransferases. Nucleic Acids Res 2005, , 33 Database: D230-232

69. Goto N, Prins P, Nakao M, Bonnal R, Aerts J, Katayama T: BioRuby: bioinformatics software for the Ruby programming language. Bioinformatics 2010, 26(20):2617-2619.

70. Barrett T, Troup DB, Wilhite SE, Ledoux P, Rudnev D, Evangelista C, Kim IF, Soboleva A, Tomashevsky M, Marshall KA, et al: NCBI GEO: archive for high-throughput functional genomic data. Nucleic Acids Res 2009, , 37 Database: D885-890

71. Swarbreck D, Wilks C, Lamesch P, Berardini TZ, Garcia-Hernandez M, Foerster H, Li D, Meyer T, Muller R, Ploetz L, et al: The Arabidopsis Information Resource (TAIR): gene structure and function annotation. Nucleic Acids Res 2008, , 36 Database: D1009-1014.

72. Raissig M, Baroux C, Grossniklaus U: Regulation and flexibility of genomic imprinting during seed development. Plant Cell 2011.

doi:10.1186/1471-2229-11-113

Cite this article as: McKeown et al:: Identification of imprinted genes subject to parent-of-origin specific expression in Arabidopsis thaliana seeds. BMC Plant Biology 2011 11:113.

\section{Submit your next manuscript to BioMed Central and take full advantage of:}

- Convenient online submission

- Thorough peer review

- No space constraints or color figure charges

- Immediate publication on acceptance

- Inclusion in PubMed, CAS, Scopus and Google Scholar

- Research which is freely available for redistribution

Submit your manuscript at www.biomedcentral.com/submit
Ciomed Central 